\title{
Probing AGB nucleosynthesis via accurate Planetary Nebula abundances
}

\author{
P. Marigo ${ }^{1}$, J. Bernard-Salas ${ }^{2,3}$, S. R. Pottasch ${ }^{3}$, A. G. G. M. Tielens ${ }^{2,3}$, and P. R. Wesselius ${ }^{2,3}$ \\ 1 Dipartimento di Astronomia, Università di Padova, Vicolo dell'Osservatorio 2, 35122 Padova, Italy \\ 2 SRON National Institute for Space Research, PO Box 800, NL 9700 AV Groningen, The Netherlands \\ 3 Kapteyn Astronomical Institute, PO Box 800, 9700 AV Groningen, The Netherlands
}

Received 28 January 2003 / Accepted 17 June 2003

\begin{abstract}
The elemental abundances of ten planetary nebulae, derived with high accuracy including ISO and IUE spectra, are analysed with the aid of synthetic evolutionary models for the TP-AGB phase. The accuracy on the observed abundances is essential in order to make a reliable comparison with the models. The advantages of the infrared spectra in achieving this accuracy are discussed. Model prescriptions are varied until we achieve the simultaneous reproduction of all elemental features, which allows placing important constraints on the characteristic masses and nucleosynthetic processes experienced by the stellar progenitors. First of all, it is possible to separate the sample into two groups of PNe, one indicating the occurrence of only the third dredge-up during the TP-AGB phase, and the other showing also the chemical signature of hot-bottom burning. The former group is reproduced by stellar models with variable molecular opacities (see Marigo 2002), adopting initial solar metallicity, and typical efficiency of the third dredge-up, $\lambda \sim 0.3-0.4$. The latter group of PNe, with extremely high He content $(0.15 \leq \mathrm{He} / \mathrm{H} \leq 0.20)$ and marked oxygen deficiency, is consistent with original sub-solar metallicity (i.e. LMC composition). Moreover, we are able to explain quantitatively both the $\mathrm{N} / \mathrm{H}-\mathrm{He} / \mathrm{H}$ correlation and the $\mathrm{N} / \mathrm{H}-\mathrm{C} / \mathrm{H}$ anti-correlation, thus solving the discrepancy pointed out long ago by Becker \& Iben (1980). This is obtained only under the hypothesis that intermediatemass TP-AGB progenitors $\left(M \gtrsim 4.5-5.0 M_{\odot}\right)$ with LMC composition have suffered a number of very efficient, carbon-poor, dredge-up events. Finally, the neon abundances of the He-rich PNe can be recovered by invoking a significant production of ${ }^{22} \mathrm{Ne}$ during thermal pulses, which would imply a reduced role of the ${ }^{22} \mathrm{Ne}(\alpha, n)^{25} \mathrm{Mg}$ reaction as neutron source to the s-process nucleosynthesis in these stars.
\end{abstract}

Key words. stars: AGB and post-AGB - stars: evolution - stars: mass loss - planetary nebulae: general - nuclear reactions, nucleosynthesis, abundances

\section{Introduction}

Planetary Nebulae (PNe) are assumed to consist of the gas ejected via stellar winds by low- and intermediate-mass stars (having initial masses $0.9 \leq M / M_{\odot} \leq M_{\text {up }}$, with $M_{\text {up }} \sim$ 5-8 $M_{\odot}$ depending on model details) during their last evolutionary stages, the so-called Thermally Pulsing Asymptotic Giant Branch (TP-AGB) phase.

PNe offer potentially a good possibility to test the results of stellar nucleosynthesis. This can be done in a reliable way by comparing the predicted abundances of the gas ejected close to the end of the AGB phase with the observed abundances because the expelled hot gas remains unaffected by interaction with the ISM or with previous shell ejection. Furthermore the ionized gas surrounding the central star shows lines of many elements from which accurate abundances can be derived. Also by the ejection of the outer layers $\mathrm{PNe}$ contribute to the enrichment of the interstellar medium (ISM) and therefore,

Send offprint requests to: $\mathrm{P}$. Marigo,

e-mail: marigo@pd.astro.it a knowledge of these processes are essential to better understand the chemical composition of the Galaxy.

PN elemental abundances represent the cumulative record of all nucleosynthetic and mixing processes that may have changed the original composition of the gas since the epoch of stellar formation. In fact, stellar evolution models predict the occurrence of several episodes in which the envelope chemical composition is altered by mixing with nuclear products synthesised in inner regions and brought up to the surface by convective motions (e.g. Iben \& Renzini 1983; Forestini \& Charbonnel 1997; Girardi et al. 2000). These dredge-up events usually take place when a star reaches its Hayashi line and develops an extended convective envelope, either during the ascent on the Red Giant Branch (RGB; the first dredge-up), or later on the early AGB (the second dredge-up). Then, the subsequent TP-AGB evolution is characterised by a rich nucleosynthesis whose products may be recurrently exposed to the surface synchronised with thermal pulses (the third dredge-up), or convected upward from the deepest envelope layers of the most massive stars (hot-bottom burning, hereinafter also HBB). 
As a consequence, the surface abundances of several elements (e.g. $\mathrm{H}, \mathrm{He}, \mathrm{C}, \mathrm{N}, \mathrm{O}, \mathrm{Ne}, \mathrm{Mg}$ ) may be significantly altered, to an extent that crucially depends on stellar parameters (i.e. mass and metallicity) various incompletely understood physical processes (e.g. convection, mass loss), and model input prescriptions (e.g. nuclear reaction rates, opacities, etc.). In this sense, the interpretation of the elemental patterns observed in PNe should give a good insight into the evolutionary and nucleosynthetic properties of the stellar progenitors, thus putting constraints on these processes.

For instance, the enrichment in $\mathrm{C}$ exhibited by some $\mathrm{PNe}$ should give a measure of the efficiency of the third dredge-up, still a matter of debate. Conversely, the deficiency in $\mathrm{C}$ shown by some $\mathrm{PNe}$ with $\mathrm{N}$ overabundance could be interpreted as the imprint of HBB, implying rather massive TP-AGB stellar progenitors (with initial masses $\geq 4 M_{\odot}$ ). The He content should measure the cumulative effect produced by the first, possibly second and third dredge-up, and HBB. The $\mathrm{O}$ abundance may help to constrain the chemical composition of the convective inter-shell developed at thermal pulses, as well as the efficiency of HBB, depending on whether this element is found to be preserved, enhanced or depleted in the nebular composition. Finally, the Ne abundance in PNe could give important information about the synthesis of this element during thermal pulses, i.e. providing an indirect estimate of the efficiency of the ${ }^{22} \mathrm{Ne}(\alpha, n)^{25} \mathrm{Mg}$ reaction with important implications for the slow-neutron capture nucleosynthesis.

In this context, the present study aims at investigating the above issues by analysing accurate determinations of elemental abundances for a sample of PNe with the aid of stellar models for low- and intermediate stars, that follow their evolution from the main sequence up to the stage of PN ejection. Particular attention is paid to modelling the TP-AGB phase to derive indications on the mass range and the metallicity of the stellar progenitors involved, and the related nucleosynthetic processes, i.e. the second and third dredge-up and HBB.

An important problem when deriving PNe abundances is the correction for unseen stages of ionization. When using optical and/or UV spectra many stages of ionization are missing and have to be inferred, making the error in the abundance determination high. This estimate for unobserved stages of ionization is done making use of Ionization Correction Factors $(I C F)$ which are mainly derived on the basis of similarities of ionization potentials or ionization models. The latter needs a very good knowledge of the stellar parameters which is often not known. When all important stages of ionization of a certain element are measured no $I C F$ is needed (or $I C F=1$ ). In the past literature $I C F$ ranging from 2-5 (and sometimes 20) are found. Many missing stages of ionization are seen in the infrared providing an important complement to the UV and optical spectra. The ICF of many elements has been drastically reduced thanks to the inclusion of the ISO (Kessler et al. 1996) data. It has certainly improved the $\mathrm{Ne}, \mathrm{Ar}, \mathrm{Cl}$ and $\mathrm{S}$ abundances and has provided information of other important stages of ionization such as $\mathrm{C}^{++}, \mathrm{O}^{3+}$ and $\mathrm{N}^{++}$. In many cases the $I C F$ is not needed and on the others is often lower than 1.5. Another important advantage is the independence of the infrared lines to the adopted electron temperature. This avoids uncertainties when the electron temperature adopted to derive the abundances is uncertain or when there are electron temperature fluctuations in the nebula. These and other advantages have been previously discussed by Beintema \& Pottasch (1999) and Bernard Salas et al. (2001).

The paper is organised as follows. Section 2 introduces the sample of ten planetary nebulae, in terms of individual characteristic like: galactic coordinates, radii, nebular fluxes in $\mathrm{H}$ and $\mathrm{He}$ II recombination lines, Zanstra temperatures and luminosities of the central nuclei. These two latter parameters locate the central stars in the Hertzsprung-Russell (HR) diagram. Section 3.1 presents the nebular elemental abundances of $\mathrm{He}$, $\mathrm{C}, \mathrm{N}, \mathrm{O}, \mathrm{Ne}, \mathrm{S}$, and $\mathrm{Ar}$, compared with the solar values, and subgrouped as a function of helium content. Section 4 outlines a summary of the main physical processes expected to alter the surface chemical composition of low- and intermediate-mass stars. Section 5 details the synthetic TP-AGB models adopted for our theoretical study, in terms of the main input parameters. The interpretative analysis of the abundance data is developed in Sect. 6. Finally, a recapitulation of the most relevant conclusions and implications in Sect. 7 closes the paper.

\section{Sample of PNe}

\subsection{General}

The sample used is biased to bright objects, in order to measure many different stages of ionization and accurately derive their abundances. The general parameters of the PNe used in this study are given in Table 1 . References for the assumed distances, magnitudes and extinction are given as footnotes in the table.

Galactic coordinates show that most of these nebulae belong to the disk (except NGC 6543 and NGC 7662) and could be descendants of young progenitors. Distances are very uncertain and great care was taken to adopt the most reliable ones from the literature. They vary between 0.8 and $2.5 \mathrm{kpc}$ and therefore are close, as would be expected for bright objects. There are different values in the literature that do not agree within the uncertainties the authors quote. Note that the $m_{\mathrm{V}}$ of He 2-111 and NGC 6302 are assumed since their central stars have never been seen. The extinction is low in most cases except for NGC 6537. In order to classify them according to their chemical composition we can investigate the $\mathrm{C} / \mathrm{O}$ ratio together with the $\mathrm{He} / \mathrm{H}$ abundance in Table 3. There are two $\mathrm{C}$-rich PNe, six O-rich PNe and two for which it is difficult to assess their nature since the $\mathrm{C} / \mathrm{O}$ ratio is (although lower than one) very close to unity (within the uncertainties). Notice that this sample contains a higher percentage of $\mathrm{PNe}$ with a high $\mathrm{He} / \mathrm{H}$ ratio than many other samples.

\subsection{HR diagram}

With the data in Table 1 and the $\mathrm{H} \beta$ and helium $\lambda 4686 \AA$ fluxes the Zanstra temperatures $\left(T_{\mathrm{Z}}\right)$, radii and luminosities have been derived (see Table 2). As pointed out by Stasińska \& Tylenda (1986) when using the Zanstra method, $T_{\mathrm{Z}}$ is over-estimated in the case of hydrogen and underestimated when using 
Table 1. Parameters of the PNe. The $m_{\mathrm{V}}$ is not corrected for extinction. References to distance, $V$ magnitude and extinction are given by superscripts $d x, m x$, and $e x$ respectively.

\begin{tabular}{|c|c|c|c|c|c|}
\hline Name & $l\left(^{\circ}\right)$ & $b\left(^{\circ}\right)$ & $d(\mathrm{kpc})$ & $m_{\mathrm{V}}(\mathrm{mag})$ & $E_{B-V}$ \\
\hline NGC $2440^{d 1, m 1, e 1}$ & 234.8 & 2.42 & 1.63 & 17.49 & 0.34 \\
\hline NGC $5315^{d 2, m 2, e 2}$ & 309.1 & -4.40 & 2.60 & 14.40 & 0.37 \\
\hline NGC $6302^{d 3, m 3, e 3}$ & 349.5 & 1.06 & 1.60 & 18.90: & 0.88 \\
\hline NGC $6445^{d 1, e 4, e 4}$ & 8.08 & 3.90 & 2.25 & 19.04 & 0.72 \\
\hline NGC $6537^{d 1, m 4, e 5}$ & 10.10 & 0.74 & 1.95 & 22.40 & 1.22 \\
\hline NGC $6543^{d 4, m 1, e 6}$ & 96.47 & 29.9 & 1.00 & 11.29 & 0.07 \\
\hline NGC $6741^{d 1, m 5, e 7}$ & 34.6 & -2.28 & 1.65 & 19.26 & 0.75 \\
\hline NGC $7027^{d 5, m 1, e 8}$ & 84.93 & -3.50 & 0.65 & 16.53 & 0.85 \\
\hline NGC $7662^{d 3, m 1, e 7}$ & 106.6 & -17.6 & $0.96^{*}$ & 14.00 & 0.12 \\
\hline He $2-111^{d 1, m 3, e 5}$ & 315.0 & -0.37 & 2.50 & 20.00: & 0.77 \\
\hline
\end{tabular}

References: ${ }^{d 1}$ Average from Acker et al. (1992); ${ }^{d 2}$ Liu et al. (2001); ${ }^{d 3}$ Terzian (1997); ${ }^{d 4}$ Reed et al. (1999); ${ }^{d 5}$ Bains et al. (2003); ${ }^{m 1}$ Ciardullo et al. (1999); ${ }^{m 2}$ Acker et al. (1992); ${ }^{m 3}$ Assumed $m_{\mathrm{V}} ;{ }^{m 4}$ Pottasch (2000); ${ }^{m 5}$ Heap et al. (1989); ${ }^{e 1}$ Bernard-Salas et al. (2002); ${ }^{e 2}$ Pottasch et al. (2002); ${ }^{e 3}$ Beintema \& Pottasch (1999); ${ }^{e 4}$ van Hoof et al. (2000); ${ }^{e 5}$ Pottasch et al. (2000); ${ }^{e 6}$ Bernard-Salas (2003a); ${ }^{e 7}$ Pottasch et al. (2001);

${ }^{e 8}$ Bernard Salas et al. (2001).

: Large error.

${ }^{*}$ Average distance by Terzian (1997).

Table 2. Radius, Zanstra temperature and luminosity of the observed PNe. $F(\mathrm{H} \beta)$ and $F(4684 \AA)$ are in units of $10^{-12} \mathrm{erg}^{-2} \mathrm{~cm}^{-1}($ not corrected for extinction). The radius is in meter.

\begin{tabular}{l|cccc|cccc}
\hline \hline \multirow{2}{*}{ Name } & \multicolumn{4}{|c|}{ Hydrogen } & \multicolumn{4}{c}{ Helium } \\
\cline { 2 - 9 } & $F(\mathrm{H} \beta)^{\dagger}$ & Radius & $\log \left(\frac{L_{*}}{L_{\odot}}\right)$ & $\log T_{\mathrm{Z}}(\mathrm{H})$ & $F(4686 \AA)^{*}$ & Radius & $\log \left(\frac{L_{*}}{L_{\odot}}\right)$ & $\log T_{\mathrm{Z}}(\mathrm{He})$ \\
\hline NGC 2440 & 31.6 & $2.2 \mathrm{E}+07$ & 2.95 & 5.25 & 19.3 & $2.1 \mathrm{E}+07$ & 3.08 & 5.29 \\
NGC 5315 & 38.1 & $2.7 \mathrm{E}+08$ & 3.34 & 4.80 & - & - & - & - \\
NGC 6302: & 29.5 & $1.7 \mathrm{E}+07$ & 3.86 & 5.52 & 16.6 & $1.7 \mathrm{E}+07$ & 3.88 & 5.53 \\
NGC 6445 & 7.60 & $2.4 \mathrm{E}+07$ & 3.19 & 5.28 & 3.40 & $2.4 \mathrm{E}+07$ & 3.19 & 5.28 \\
NGC 6537 & 2.20 & $5.8 \mathrm{E}+06$ & 3.50 & 5.67 & 3.10 & $4.6 \mathrm{E}+06$ & 4.09 & 5.87 \\
NGC 6543 & 245 & $3.6 \mathrm{E}+08$ & 2.96 & 4.64 & 14.7 & $2.8 \mathrm{E}+08$ & 3.46 & 4.82 \\
NGC 6741 & 4.40 & $1.8 \mathrm{E}+07$ & 2.69 & 5.22 & 1.60 & $1.8 \mathrm{E}+07$ & 2.69 & 5.22 \\
NGC 7027 & 75.9 & $2.7 \mathrm{E}+07$ & 3.31 & 5.29 & 31.1 & $2.8 \mathrm{E}+07$ & 3.27 & 5.28 \\
NGC 7662 & 102 & $7.8 \mathrm{E}+07$ & 2.54 & 4.87 & 17.4 & $6.8 \mathrm{E}+07$ & 2.82 & 4.97 \\
He 2-111: & 0.98 & $2.5 \mathrm{E}+07$ & 2.37 & 5.08 & 0.89 & $2.0 \mathrm{E}+07$ & 2.82 & 5.24 \\
\hline
\end{tabular}

: Large error in the radius, temperature and luminosity.

$\dagger$ From the same references as the extinction in Table 1.

${ }^{*}$ From Acker et al. (1992).

helium. This is because the Zanstra method assumes that energies above $54.4 \mathrm{eV}$ are only absorbed by helium. This is not completely true. In addition recombination of $\mathrm{He}^{2+}$ sometimes produces more than one photon which can ionize hydrogen and the proportion of stellar photons with energies above 54.4 increases with $T_{\text {eff. }}$. Both $T_{\mathrm{Z}}(\mathrm{He})$ and $T_{\mathrm{Z}}(\mathrm{H})$ yield the same results for most PNe. $T_{Z}(\mathrm{H})$ fails when the nebula is thin and some photons escape. In the case of a thick nebula both methods should yield the same result, but this is tricky because a nebula can be thick in the torus and thin in the poles. For all those reasons the $T_{\mathrm{Z}}(\mathrm{He})$ was preferred over $T_{\mathrm{Z}}(\mathrm{H})$.
These results are shown in Fig. 1. For NGC 5315 no helium line is detected so that results using $T_{\mathrm{Z}}(\mathrm{H})$ have been plotted. The evolutionary tracks of Vassiliadis \& Wood (1994) are related to the core mass rather than the initial mass. Stars with different initial mass and different mass loss functions can lead to the same core mass.

The majority of PNe are within the range of temperature and luminosity of the theoretical evolutionary tracks. Most are in the last stage of the PNe phase. NGC 6537 lies outside and it must be noticed that using $T_{\mathrm{Z}}(\mathrm{H})$ makes this object closer to NGC 6302. While this figure may provide some insight in 
Table 3. PNe abundances ${ }^{\natural}$ w.r.t. hydrogen in number. The number between parenthesis $(x)$ stands for $10^{x}$. Abundance reference values for the Sun, Orion, and LMC are also included.

\begin{tabular}{|c|c|c|c|c|c|c|c|}
\hline Name & Helium & Carbon(-4) & Nitrogen $(-4)$ & Oxygen $(-4)$ & Neon $(-4)$ & Sulfur $(-5)$ & $\operatorname{Argon}(-6)$ \\
\hline NGC 2440 & 0.119 & 7.2 & 4.4 & 3.8 & 1.1 & 0.5 & 3.2 \\
\hline NGC 5315 & 0.124 & 4.4 & 4.6 & 5.2 & 1.6 & 1.2 & 4.6 \\
\hline NGC 6302 & 0.170 & 0.6 & 2.9 & 2.3 & 2.2 & 0.8 & 6.0 \\
\hline NGC 6445 & 0.140 & 6.0 & 2.4 & 7.4 & 2.0 & 0.8 & 3.8 \\
\hline NGC 6537 & 0.149 & 1.8 & 4.5 & 1.8 & 1.7 & 1.1 & 4.1 \\
\hline NGC 6543 & 0.118 & 2.5 & 2.3 & 5.5 & 1.9 & 1.3 & 4.2 \\
\hline NGC 6741 & 0.111 & 6.4 & 2.8 & 6.6 & 1.8 & 1.1 & 4.9 \\
\hline NGC 7027 & 0.106 & 5.2 & 1.5 & 4.1 & 1.0 & 0.9 & 2.3 \\
\hline NGC 7662 & 0.088 & 3.6 & 0.7 & 4.2 & 0.6 & 0.7 & 2.1 \\
\hline He 2-111 & 0.185 & 1.1 & 3.0 & 2.7 & 1.6 & 1.5 & 5.5 \\
\hline Sun* & 0.100 & 3.55 & 0.93 & 4.9 & 1.2 & 1.86 & 3.6 \\
\hline Orion $^{\dagger}$ & 0.098 & 2.5 & 0.60 & 4.3 & 0.78 & 1.5 & 6.3 \\
\hline$<\mathrm{LMC}>^{\sharp}$ & 0.089 & 1.10 & 0.14 & 2.24 & 0.41 & 0.65 & 1.9 \\
\hline
\end{tabular}

${ }^{\natural}$ See Sect. 3.1 for references.

* Grevesse \& Sauval (1998) and Anders \& Grevesse (1989) except the oxygen abundance which was taken from Allende Prieto et al. (2001).

† Esteban et al. (1998).

$\sharp$ From Dopita et al. (1997).

core mass and time evolution of these objects, the many uncertainties (especially in distance) should be born in mind.

\section{Accurate abundances of ISO-observed PNe}

\subsection{Abundances}

Accurate abundances are needed in order to make a reliable comparison with theoretical models. For this purpose a sample of ten PNe was selected in which precise abundances using ISO data have been derived. The references for the abundances are as follows: NGC $2440 \rightarrow$ Bernard Salas et al. (2002); NGC $5315 \rightarrow$ Pottasch et al. (2002); NGC 6302 $\rightarrow$ Pottasch \& Beintema (1999); NGC 6445 $\rightarrow$ van Hoof et al. (2000); NGC 6537 and He 2-111 $\rightarrow$ Pottasch et al. (2000); NGC $6543 \rightarrow$ Bernard-Salas et al. (2003a); NGC 6741 and NGC 7662 $\rightarrow$ Pottasch et al. (2001); NGC 7027 $\rightarrow$ Bernard Salas et al. (2001) and Bernard Salas et al. (2003b).

It should be noticed that NGC 6302, NGC 6537 and He 2-111 are among those $\mathrm{PNe}$ with a hot central star, a strong bipolar morphology, and in which high velocity shocks are present. The presence of the latter can affect the abundance composition which has been derived assuming that the ionization is produced by the hot central star. For He 2-111 the velocity of the shocks have been estimated by Meaburn \& Walsh (1989) to be $\sim 380 \mathrm{~km} \mathrm{~s}^{-1}$. They show that these high velocity shocks are localized in the outermost parts of the bi-polar lobes (which are less dense). They conclude that photoionization by a hot star is the most plausible dominant process in the core and dense disk. Since these shocks affect the less denser regions the effect that they might have on the abundance determination is very small and can be neglected.
NGC 6302 has velocity shocks of $\sim 500 \mathrm{~km} \mathrm{~s}^{-1}$ (Lame \& Ferland 1991). They fit photoionization models to the spectrum which indicate that only very high stages of ionization, such as $\mathrm{Si}^{6}+(\mathrm{IP} 167 \mathrm{eV})$, are produced by shocks. These high stages of ionization have negligible weight on the overall abundance which is therefore not affected by the presence of the shocks. The speed of the shocks in NGC 6537 is $\sim 300 \mathrm{~km} \mathrm{~s}^{-1}$ (Corradi \& Schwarz 1993). Hyung (1999) studied this specific problem of the source of the nebular emission arising either by the hot central star or by shock heating, with the help of photoionization models. He concludes that the radiation of the hot central star is responsible for the emission. In summary, the high velocity shocks present in these $\mathrm{PNe}$ do not play a role in the abundance determination.

The error in the abundances in Table 3 is about 20 to $30 \%$. These errors only include the uncertainty in the intensities of the lines used to derive the abundances. Other errors, e.g. using Ionization Correction Factor (ICF) to derive abundances or the effect of uncertainties in the atomic parameters (especially the collisional strengths) are difficult to quantify. The abundances shown in Table 3 have been derived using an ICF near unity. Often all important stages of ionization are observed, in which case no error from the $I C F$ is involved. The abundances have been obtained using the most recent results available for the collisional strengths (mainly from the IRON project) so that these uncertainties are much reduced. A quantitative idea of the errors on the abundance can also be inferred by looking at the sulfur and argon abundances in Fig. 2. Sulfur and argon are not supposed to vary in the course of evolution, and as can be seen from the figure, they are within $30 \%$. This strongly suggests that the abundance error derived from the intensity of the lines is the main contributor to the total error. Therefore $30 \%$ 


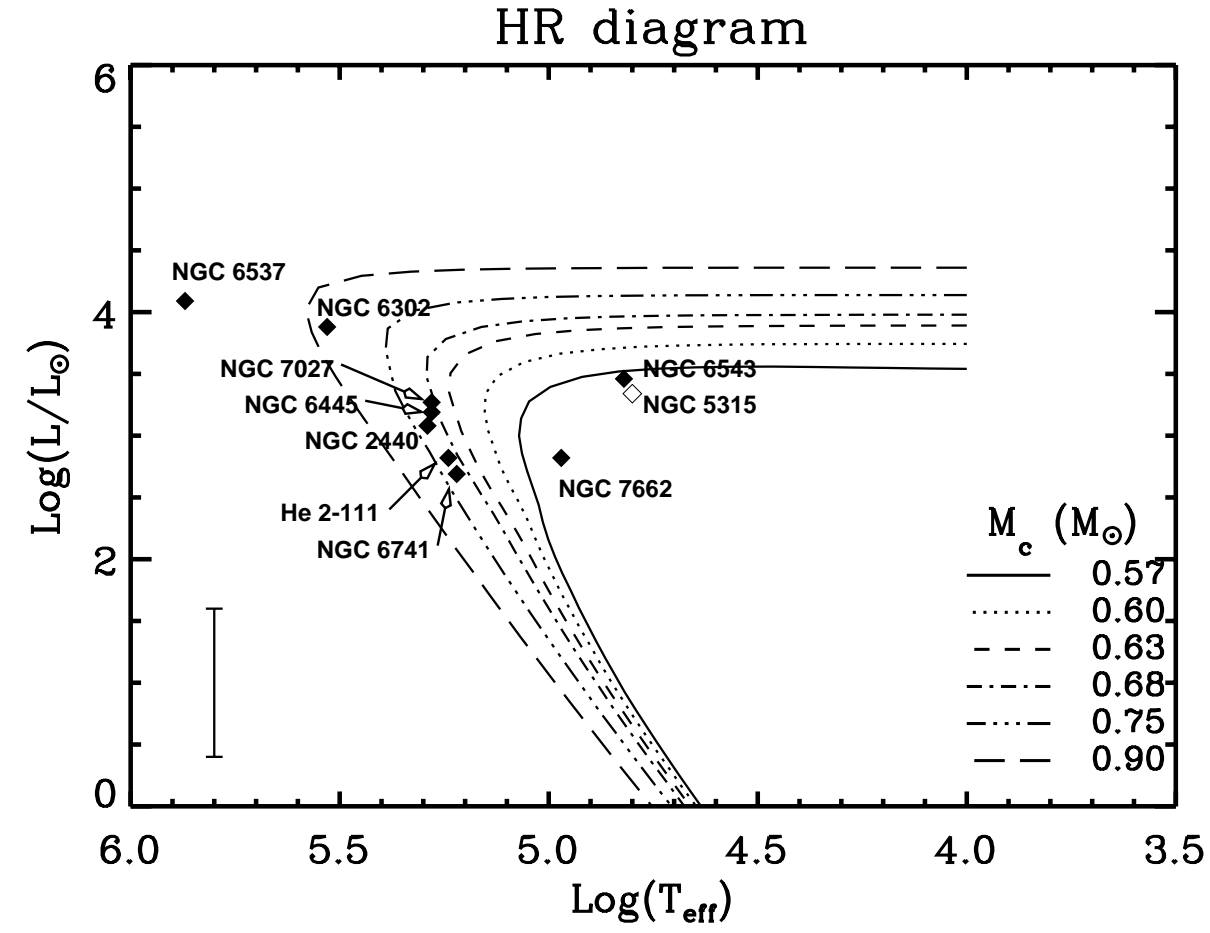

Fig. 1. HR diagram for the PNe of the sample (diamonds). The $T_{\text {eff }}$ have been derived with the Zanstra method using the helium lines except for NGC 5315 (open diamond). The Post-AGB evolutionary tracks from Vassiliadis \& Wood (1994) for $Z=0.016$ are also plotted for different core masses, indicated in the lower-right corner of the figure. In the lower-left the uncertainty in the luminosity due to the error of a factor two in the distance is shown.

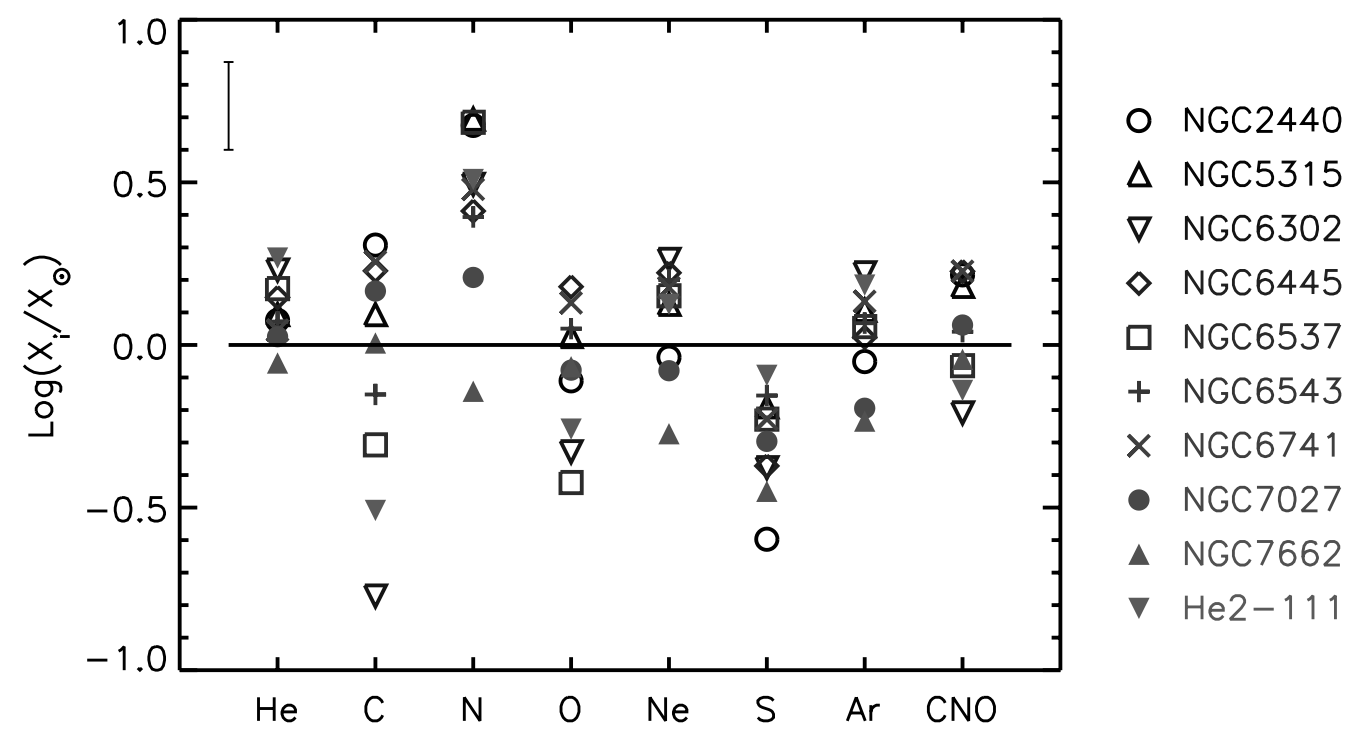

Fig. 2. Observational abundances with respect to Solar. In the upper-left corner a typical error bar for all elements except helium is plotted. The solid line represents abundances equal to solar abundances.

is a good estimate of the abundance error and has been assumed in this work for all nebulae except for the nitrogen abundance of NGC 6543 which has an error 50\%. This is because the main contribution to the nitrogen abundance comes from the $\mathrm{N}^{++}$whose ionic abundance is derived either with the $57.3 \mu \mathrm{m}$ or $1750 \AA$ lines. The former is density dependent and the second temperature dependent, increasing therefore the error. The error in the helium abundance is around 5\% except, again, for NGC 6543 where it is $7-8 \%$.

\subsection{Comparison with Solar abundances}

The PNe abundances (Table 3) are shown in Fig. 2 with respect to the solar. The typical error bar applies to all elements except helium for which the error is 6 times smaller. This comparison is important because in principle one might expect that the progenitor stars of these PNe have evolved from a solar metallicity. Therefore, primary elements that do not change in the course 
of evolution should lie close to the solar line, elements that are destroyed or produced should lie below or above.

An inspection of the figure leads to several conclusions. NGC 7662 shows low abundances for all elements. It is then suspected that the progenitor mass of this PNe was low and that it did not experience much change in the course of evolution.

All other PNe show a He enhancement, which should be expected as He is brought to the surface in the different dredgeup episodes. Four PNe show a decrease in carbon, especially NGC 6302 and He 2-111. The remaining PNe show solar or enhanced carbon. All PNe (except NGC 7662) show an increase of nitrogen. The oxygen abundance is close to solar for all PNe except three. The exceptions are NGC 6302, NGC 6537 and He 2-111 which show a clear decrease. It should be noticed that the solar abundance adopted in this work is that of Allende Prieto et al. (2001). If the value of Grevesse \& Sauval (1998) had been adopted all PNe would lie below the solar value.

Within the errors all neon abundances except that of NGC 7662 agree with solar. Of all elements neon is probably the best determined and it is likely that the error is somewhat smaller than $30 \%$. In this sense it is interesting to see how PNe with higher neon abundances are clumped at around 0.15 and the three with lower values also have lower helium abundance of the sample.

The remaining elements, sulfur and argon, are supposed to remain unchanged in the course of evolution and therefore should be close to solar. The argon abundance is compatible with this but the sulfur abundance is lower than solar.

\section{Nucleosynthesis in low- and intermediate-mass stars}

We will recall the main nucleosynthetic and convective mixing events that may possibly alter the surface chemical composition of a low-/intermediate-mass star in the course of its evolution. According to the classical scenario ${ }^{1}$ four processes are important (see e.g. Forestini \& Charbonnel 1997 for a recent extended analysis), namely:

The first dredge-up. At the base of the RGB the outer convective envelope reaches regions of partial hydrogen burning (CN cycle). As a consequence, the surface abundance of ${ }^{4} \mathrm{He}$ is increased (and that of $\mathrm{H}$ depleted), ${ }^{14} \mathrm{~N}$ and ${ }^{13} \mathrm{C}$ are enhanced at the expense of ${ }^{12} \mathrm{C}$, while ${ }^{16} \mathrm{O}$ remains almost unchanged.

The second dredge-up. This occurs in stars initially more massive than 3-5 $M_{\odot}$ (depending on composition) during the early AGB phase. The convective envelope penetrates into the helium core (the H-burning shell is extinguished) so that the surface abundances of ${ }^{4} \mathrm{He}$ and ${ }^{14} \mathrm{~N}$ increase, while those of ${ }^{12} \mathrm{C},{ }^{13} \mathrm{C}$ and ${ }^{16} \mathrm{O}$ decrease.

\footnotetext{
1 We do not consider here any additional extra-mixing process, such as the one invoked to explain the observed abundance anomalies in low-mass RGB stars (see e.g. Charbonnel 1995).
}

The third dredge-up. This takes place during the TP-AGB evolution in stars more massive than $\approx 1.5 M_{\odot}$ for solar composition, starting at lower masses for lower metallicities (see Marigo et al. 1999). It actually consists of several mixing episodes occurring at thermal pulses during which significant amounts of ${ }^{4} \mathrm{He}$ and ${ }^{12} \mathrm{C}$, and smaller quantities of other newly-synthesized products (e.g. ${ }^{16} \mathrm{O},{ }^{22} \mathrm{Ne},{ }^{25} \mathrm{Mg}$, s-process elements) are convected to the surface.

Hot bottom burning. This occurs in the most massive and luminous AGB stars (with initial masses $M \gtrsim 4-4.5 M_{\odot}$, depending on metallicity). The convective envelope penetrates deeply into the hydrogen-burning shell, and the $\mathrm{CN}$-cycle nucleosynthesis actually occurs in the deepest envelope layers of the star. As a consequence, besides the synthesis of new helium, ${ }^{12} \mathrm{C}$ is first converted into ${ }^{13} \mathrm{C}$ and then into ${ }^{14} \mathrm{~N}$. In the case of high temperatures and after a sufficiently long time, the ON cycle can also be activated, so that ${ }^{16} \mathrm{O}$ is burned into ${ }^{14} \mathrm{~N}$.

It should be remarked that the third dredge-up and hotbottom burning are the processes that are expected to produce the most significant changes in $\mathrm{CNO}$ and He surface abundances, being affected at the same time by the largest uncertainties in the theory of stellar evolution. This latter point motivates the adoption of free parameters (e.g. the dredge-up efficiency) to describe these processes in synthetic TP-AGB models, that are discussed next.

\section{Synthetic TP-AGB calculations}

In order to interpret the abundance data reported in Sect. 3.1, synthetic evolutionary calculations of the TP-AGB phase have been carried out with the aid of the model developed by Marigo et al. (1996, 1998, 1999), Marigo (1998, 2001, 2002), to whom we refer for all details.

We briefly recall that the initial conditions at the first thermal pulse are extracted from full stellar models with convective overshooting by Girardi et al. (2000). The considered mass interval $\left(0.7-5.0 M_{\odot}\right)$ covers the whole class of low- and intermediate-mass stars that develop an electron-degenerate $\mathrm{C}-\mathrm{O}$ core at the end of the core He-burning phase. In these models the initial chemical composition is taken from Anders \& Grevesse (1989) for solar metallicity $(Z=0.019)$, while for other metallicities solar-scaled abundances are assumed.

The Girardi et al. (2000) models predict the changes (if any) in the surface chemical composition occurring prior to the TP-AGB phase by the first dredge-up during the first settling on the Red Giant Branch, and by the second dredge-up in stars of intermediate mass (say $M>3.5-4 M_{\odot}$ ) during the Early AGB phase.

The subsequent TP-AGB evolution is calculated from the first thermal pulse up to the complete ejection of the envelope by stellar winds. Mass loss is included according to the Vassiliadis \& Wood's (1993) formalism. The TP-AGB model predicts the changes in the surface chemical composition caused by the third dredge-up and HBB. The third dredgeup is parametrized as a function of the efficiency, $\lambda$, and the minimum temperature at the base of the convective envelope, 
Table 4. Assumptions adopted in the TP-AGB synthetic calculations discussed in the paper.

\begin{tabular}{|c|c|c|c|c|c|c|c|}
\hline & & & 3rd D-up efficiency & Inter & shell compc & sition & Initial oxygen abundance \\
\hline Models (ref.) & $Z$ & Opacity $^{1}$ & $\lambda^{2}$ & $X_{\mathrm{csh}}\left({ }^{12} \mathrm{C}\right)$ & $X_{\mathrm{csh}}\left({ }^{16} \mathrm{O}\right)$ & $X_{\mathrm{csh}}\left({ }^{4} \mathrm{He}\right)$ & $X_{0}(\mathrm{O})^{3}$ \\
\hline A) Figs. 3, 9 & 0.019 & $\kappa_{\mathrm{fix}}$ & const. 0.50 & 0.220 & 0.020 & 0.760 & $\mathrm{O}_{\odot}$ High \\
\hline B) Fig. 6 & 0.019 & $\kappa_{\mathrm{var}}$ & const. 0.50 & 0.220 & 0.020 & 0.760 & $\mathrm{O}_{\odot} \mathrm{High}$ \\
\hline C) Fig. 6 & 0.019 & $\kappa_{\mathrm{var}}$ & var. 0.457 & 0.220 & 0.020 & 0.760 & $\mathrm{O}_{\odot}$ High \\
\hline D) Figs. 6, 11, 12 & 0.019 & $\kappa_{\mathrm{var}}$ & var. 0.457 & 0.220 & 0.020 & 0.760 & $\mathrm{O}_{\odot}$ Low \\
\hline E) Figs. 6, 11, 12 & 0.019 & $\kappa_{\mathrm{var}}$ & var. 0.30 & 0.220 & 0.020 & 0.760 & $\mathrm{O}_{\odot}$ Low \\
\hline F) Figs. 9, 11, 12 & 0.019 & $\kappa_{\mathrm{var}}$ & var. $0.88,0.96$ & 0.050 & 0.005 & 0.945 & $\mathrm{O}_{\odot}$ Low \\
\hline G) Fig. 10 & 0.008 & $\kappa_{\mathrm{fix}}$ & const. 0.50 & 0.220 & 0.020 & 0.760 & $\left(Z / Z_{\odot}\right) \times\left(\mathrm{O}_{\odot} \mathrm{High}\right)$ \\
\hline H) Fig. 10 & 0.008 & $\kappa_{\mathrm{fix}}$ & const. 0.90 & 0.220 & 0.020 & 0.760 & $\left(\mathrm{Z} / \mathrm{Z}_{\odot}\right) \times\left(\mathrm{O}_{\odot} \mathrm{High}\right)$ \\
\hline I) Fig. 10 & 0.008 & $\kappa_{\mathrm{var}}$ & const. 0.90 & 0.220 & 0.020 & 0.760 & $\left(\mathrm{Z} / \mathrm{Z}_{\odot}\right) \times\left(\mathrm{O}_{\odot} \mathrm{High}\right)$ \\
\hline J) Figs. 10, 11, 12 & 0.008 & $\kappa_{\mathrm{var}}$ & const. 0.90 & 0.030 & 0.001 & 0.969 & $\left(\mathrm{Z} / \mathrm{Z}_{\odot}\right) \times\left(\mathrm{O}_{\odot} \mathrm{High}\right)$ \\
\hline K) Figs. 10, 11, 12 & 0.008 & $\kappa_{\mathrm{var}}$ & const. 0.90 & 0.010 & 0.001 & 0.999 & $\left(\mathrm{Z} / \mathrm{Z}_{\odot}\right) \times\left(\mathrm{O}_{\odot} \mathrm{High}\right)$ \\
\hline
\end{tabular}

1 " $\kappa_{\mathrm{fix}}$ " corresponds to solar-scaled molecular opacities by Alexander \& Ferguson (1994);

" $\kappa_{\mathrm{var}}$ " denotes the variable molecular opacities as calculated according to Marigo (2002).

2 "const. value" means that the same constant value of $\lambda$ is adopted at each dredge-up episode;

"var. value" means that $\lambda$ is made vary up to a maximum value, according to the analytic recipe by Karakas et al. (2002, their Eq. (7)).

3 " $\mathrm{O}_{\odot}$ High" refers the determination of the oxygen abundance for the Sun by Anders \& Grevesse (1989);

"O $\mathrm{O}_{\odot}$ Low" refers to the recent determination of the oxygen abundance for the Sun by Allende Prieto et al. (2001).

Other assumptions common to all TP-AGB models are:

$\log T_{\mathrm{b}}^{\text {dred }}=6.4$ following Marigo et al. (1999);

Mixing-length parameter $\alpha=\Lambda / H_{\mathrm{p}}=1.68$ (where $H_{\mathrm{p}}$ denotes the pressure-scale height, and $\Lambda$ is the mixing-length) following Girardi et al. (2000), unless otherwise specified;

Nuclear reaction rates from Caughlan \& Fowler (1988).

$T_{\mathrm{b}}^{\text {dred }}$, required for dredge-up to occur (Marigo et al. 1999, see also Sect. 5.1). The process of HBB - expected to take place in the most massive and luminous AGB stars $\left(\gtrsim 4.5 M_{\odot}\right.$ depending on metallicity) - is followed in detail with the aid of a complete envelope model including the main nuclear reactions of the CNO cycle (Marigo et al. 1998; Marigo 1998).

Recently Marigo (2002) has introduced a major novelty in the TP-AGB model, that is the replacement of fixed solar-scaled molecular opacities - commonly adopted in most AGB evolution codes $\left(k_{\mathrm{fix}}\right)$ - with variable molecular opacities $\left(k_{\mathrm{var}}\right)$ which are consistently coupled with the actual elemental abundances of the outer stellar layers. The impact of this new prescription on the evolution of AGB stars is significant and consequently, as will be shown below, it importantly affects the predictions of the PN elemental abundances.

For the sake of clarity, in the following we will provide an outline of the main input assumptions adopted in our TP-AGB calculations (Sect. 5.1 and Table 4).

\subsection{Nucleosynthesis and mixing assumptions of the TP-AGB model}

The treatment of the third dredge-up in the TP-AGB model is characterized by:

- A temperature criterion to establish whether a dredgeup episode does or does not occur. It is based on the parameter $T_{\mathrm{b}}^{\mathrm{dred}}$, that corresponds to the minimum temperature - at the base of the convective envelope at the stage of post-flash luminosity maximum - required for dredgeup to take place. In practice a procedure, based on envelope integrations, allows one to determine the onset of the third dredge-up, that is the minimum core mass $M_{\mathrm{c}}^{\mathrm{min}}$, and luminosity at the first mixing episode. More details can be found in Marigo et al. (1999; see also Wood 1981; Boothroyd \& Sackmann 1988; Karakas et al. 2002).

- The efficiency $\lambda=\Delta M_{\text {dred }} / \Delta M_{\mathrm{c}}$. It is defined as the fraction of the core mass increment, $\Delta M_{\mathrm{c}}$, over a quiescent interpulse period, that is dredged-up to the surface at the next thermal pulse (corresponding to a mass $\Delta M_{\text {dred }}$ ).

- The composition of the convective inter-shell (in terms of the elemental abundances $X_{\mathrm{csh}, i}$ ) developed at thermal pulses, i.e. of the dredged-up material. We essentially specify the abundances of helium $X_{\mathrm{csh}}\left({ }^{4} \mathrm{He}\right)$, carbon $X_{\mathrm{csh}}\left({ }^{12} \mathrm{C}\right)$, and oxygen $X_{\mathrm{csh}}\left({ }^{12} \mathrm{O}\right)$ (see Table 4$)$. We recall that detailed calculations of thermal pulses indicate a typical intershell composition $\left[X_{\mathrm{csh}}\left({ }^{4} \mathrm{He}\right) \sim 0.76 ; X_{\mathrm{csh}}\left({ }^{12} \mathrm{C}\right) \sim 0.22\right.$; $X_{\mathrm{csh}}\left({ }^{16} \mathrm{O}\right) \sim 0.02$ ] (see e.g. Boothroyd \& Sackmann 1988; Forestini \& Charbonnel 1997). However, these results may drastically change with the inclusion of extended overshooting from convective boundaries (i.e. $X_{\mathrm{csh}}\left({ }^{12} \mathrm{C}\right) \sim 0.50$; $X_{\mathrm{csh}}\left({ }^{16} \mathrm{O}\right) \sim 0.25$ according to Herwig et al. 1997), or in the most massive TP-AGB models with deep dredge-up penetration (i.e. Vassiliadis \& Wood 1993; Frost et al. 1998). 
This latter possibility, relevant to our analysis, is discussed in Sect. 6.4.2.

In addition to ${ }^{4} \mathrm{He},{ }^{12} \mathrm{C}$, and ${ }^{16} \mathrm{O}$, we account for the possible production of ${ }^{22} \mathrm{Ne}$, via the chain of reactions ${ }^{14} \mathrm{~N}(\alpha, \gamma)$ ${ }^{18} \mathrm{~F}\left(\beta^{+}, \nu\right){ }^{18} \mathrm{O}(\alpha, \gamma){ }^{22} \mathrm{Ne}$ (Iben \& Renzini 1983). Then a certain amount of ${ }^{22} \mathrm{Ne}$ may be burned via the neutron source reaction ${ }^{22} \mathrm{Ne}(\alpha, n){ }^{25} \mathrm{Mg}$. The efficiency of this nuclear step strongly depends on the maximum temperature achieved at the bottom of the inter-shell developed at thermal pulses, being marginal for $T<3 \times 10^{8} \mathrm{~K}$ (about $1 \%$ of ${ }^{22} \mathrm{Ne}$ is converted into ${ }^{25} \mathrm{Mg}$ ), while becoming more and more important at higher temperatures (Busso et al. 1999). In our study, we parameterize the $\mathrm{Ne}$ and $\mathrm{Mg}$ production assuming that the abundances in the convective intershell are given by (see also Marigo et al. 1996):

$$
\begin{aligned}
& X_{\mathrm{csh}}\left({ }^{22} \mathrm{Ne}\right)=X_{\mathrm{e}}\left({ }^{22} \mathrm{Ne}\right)+F \times \frac{22}{14} \times X_{\mathrm{Hsh}}\left({ }^{14} \mathrm{~N}\right) \\
& X_{\mathrm{csh}}\left({ }^{25} \mathrm{Mg}\right)=X_{\mathrm{e}}\left({ }^{25} \mathrm{Mg}\right)+(1-F) \times \frac{25}{14} \times X_{\mathrm{Hsh}}\left({ }^{14} \mathrm{~N}\right)
\end{aligned}
$$

where $X_{\mathrm{e}}$ refers to the envelope abundance before the dredge-up, and $F$ represents the degree of efficiency of the ${ }^{22} \mathrm{Ne}$ production, that is clearly complementary to that of ${ }^{25} \mathrm{Mg}(1-F)$. In most of our calculations we assume $F=$ 0.99 , that means allowing the chain of $\alpha$-capture reactions to proceed from ${ }^{14} \mathrm{~N}$ to ${ }^{22} \mathrm{Ne}$, with essentially no further production of ${ }^{25} \mathrm{Mg}$. This point is discussed in Sect. 6.4.3. In the above equations $X_{\mathrm{Hsh}}\left({ }^{14} \mathrm{~N}\right)$ denotes the nitrogen abundance left by the H-burning shell in the underlying inter-shell region, just before the occurrence of the pulse. It is estimated with

$$
\begin{aligned}
& X_{\mathrm{Hsh}}\left({ }^{14} \mathrm{~N}\right)=14 \times\left[\frac{X_{\mathrm{e}}\left({ }^{12} \mathrm{C}\right)}{12}+\frac{X_{\mathrm{e}}\left({ }^{13} \mathrm{C}\right)}{13}+\frac{X_{\mathrm{e}}\left({ }^{14} \mathrm{~N}\right)}{14}\right. \\
& \left.+\frac{X_{\mathrm{e}}\left({ }^{15} \mathrm{~N}\right)}{15}+\frac{X_{\mathrm{e}}\left({ }^{16} \mathrm{O}\right)}{16}+\frac{X_{\mathrm{e}}\left({ }^{17} \mathrm{O}\right)}{17}+\frac{X_{\mathrm{e}}\left({ }^{18} \mathrm{O}\right)}{18}\right],
\end{aligned}
$$

i.e. we assume that all $\mathrm{CNO}$ nuclei are converted in ${ }^{14} \mathrm{~N}$, which is a good approximation when the CNO-cycle operates under equilibrium conditions. In this way we account for the possible primary component of ${ }^{14} \mathrm{~N}$ in the intershell, which is produced every time some freshly dredgedup ${ }^{12} \mathrm{C}$ in the envelope is engulfed by the $\mathrm{H}$-burning shell during the quiescent inter-pulse evolution.

As a consequence, the resulting $X_{\mathrm{csh}}\left({ }^{22} \mathrm{Ne}\right)$, synthesized via the chain of Eq. (1), contains a primary component, which can also be injected into the surface chemical composition through the dredge-up. We note that ${ }^{22} \mathrm{Ne}$ is expected to be purely secondary in low-mass stars that do not experience the third dredge-up.

The reader can find more details about model prescriptions in Table 4.

\section{Comparison between models and observations}

We will now perform an analysis of the observed PN elemental abundances with the aid of the TP-AGB models just described. The basic idea is to constrain the model parameters so as to reproduce the observed data, and hence derive indications on the evolution and nucleosynthesis of AGB stellar progenitors. The chemical elements under consideration are $\mathrm{He}, \mathrm{C}, \mathrm{N}, \mathrm{O}$ and $\mathrm{Ne}$.

\subsection{The starting point: TP-AGB models with solar-scaled molecular opacities}

First "old" predictions of PN abundances (Marigo 2001) are considered; see Fig. 3. In those models with an initially solar composition the dredge-up parameters $\left(\lambda\right.$ and $\left.T_{\mathrm{b}}^{\text {dred }}\right)$ were calibrated to reproduce the observed carbon star luminosity functions in both Magellanic Clouds (Marigo et al. 1999). Moreover, envelope integrations were carried out using fixed solar-scaled molecular opacities ( $\kappa_{\mathrm{fix}}$; see Sect. 5 ).

By inspecting Fig. 3 we note that, though a general agreement is found between measured and predicted abundances with respect to nitrogen and neon abundances, three main discrepancy points occur:

1. A sizeable overproduction of carbon by models, up to a factor of 3-5;

2. The lack of extremely He-rich models, with $0.15<\mathrm{He} / \mathrm{H} \leq 0.20$

3. A general overabundance of oxygen in all models, amounting up to a factor of 3 .

While the first two aspects are probably related to the nucleosynthetic assumptions in the TP-AGB models, the third one could also reflect our choice of oxygen abundance in the solar mixture (Anders \& Grevesse 1989), which has recently been subject of major revision (Allende Prieto et al. 2001). The aim of the following analysis is to single out the main causes of the disagreement and possibly to remove it by proper changes in the model assumptions.

\subsection{Sub-grouping of the ISO sample and comparison with other PNe data}

Before starting the interpretative analysis, it is worth noticing that the PNe data (see Fig. 3) seem to segregate in two different groups in the observational abundance plots - one at lower $(\mathrm{He} / \mathrm{H} \leq 0.14)$ and the other at higher helium content $(\mathrm{He} / \mathrm{H}>$ 0.14 ) -, and their chemical patterns for carbon and nitrogen already suggest likely different mass ranges for the progenitors (i.e. low- and intermediate-mass stars respectively).

In particular, there are three PNe, He 2-111, NGC 6302 and NGC 6537 that clearly show the highest helium abundance $(\mathrm{He} / \mathrm{H}>0.14)$ together with the lowest carbon and oxygen abundances. To this respect we recall that it was recognized a long time ago (Becker \& Iben 1980) that the observed $\mathrm{N} / \mathrm{O}-\mathrm{He} / \mathrm{H}$ correlation and the $\mathrm{C} / \mathrm{O}-\mathrm{N} / \mathrm{O}$ anti-correlation - characterising the He-rich $\mathrm{PNe}$ - present a problem for models of nucleosynthesis on the AGB. This will be discuss in Sect. 6.4.

Comparison with literature data on PN elemental abundances provides further support for two separate groups of $\mathrm{PNe}$ according to their helium content, "normal" or high. Figure 5 compares our observed abundances with data from 


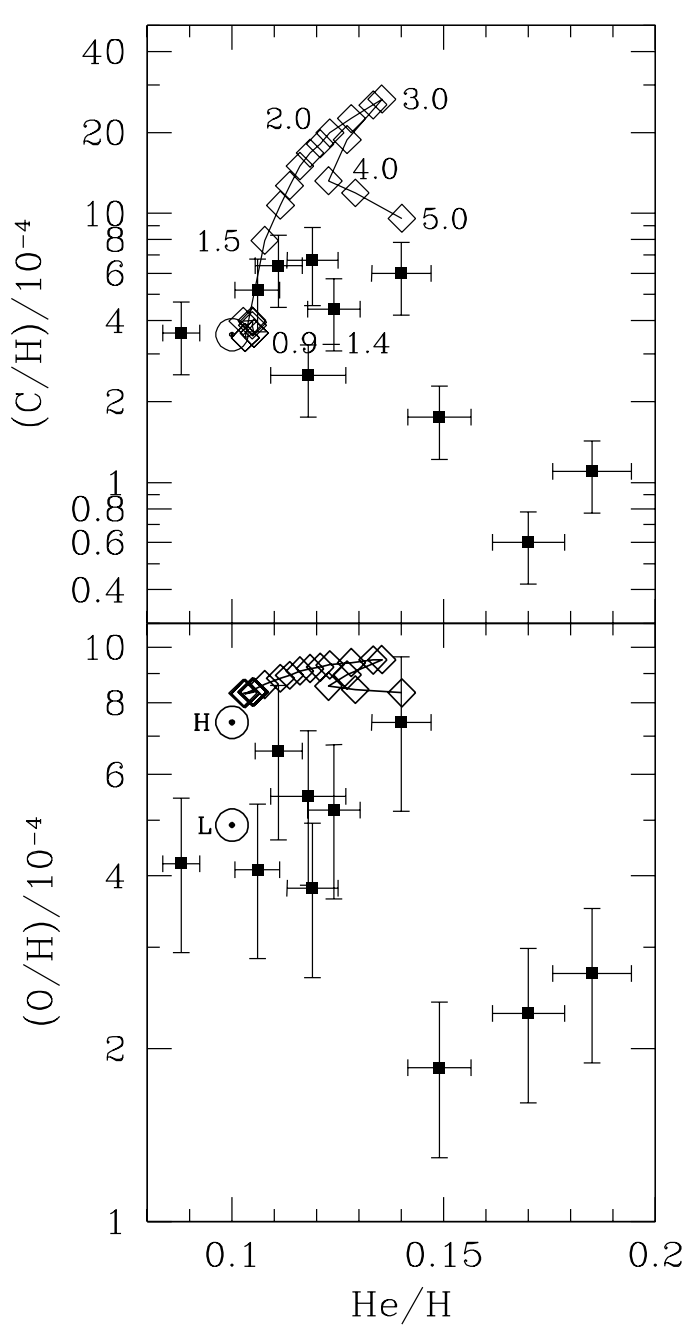

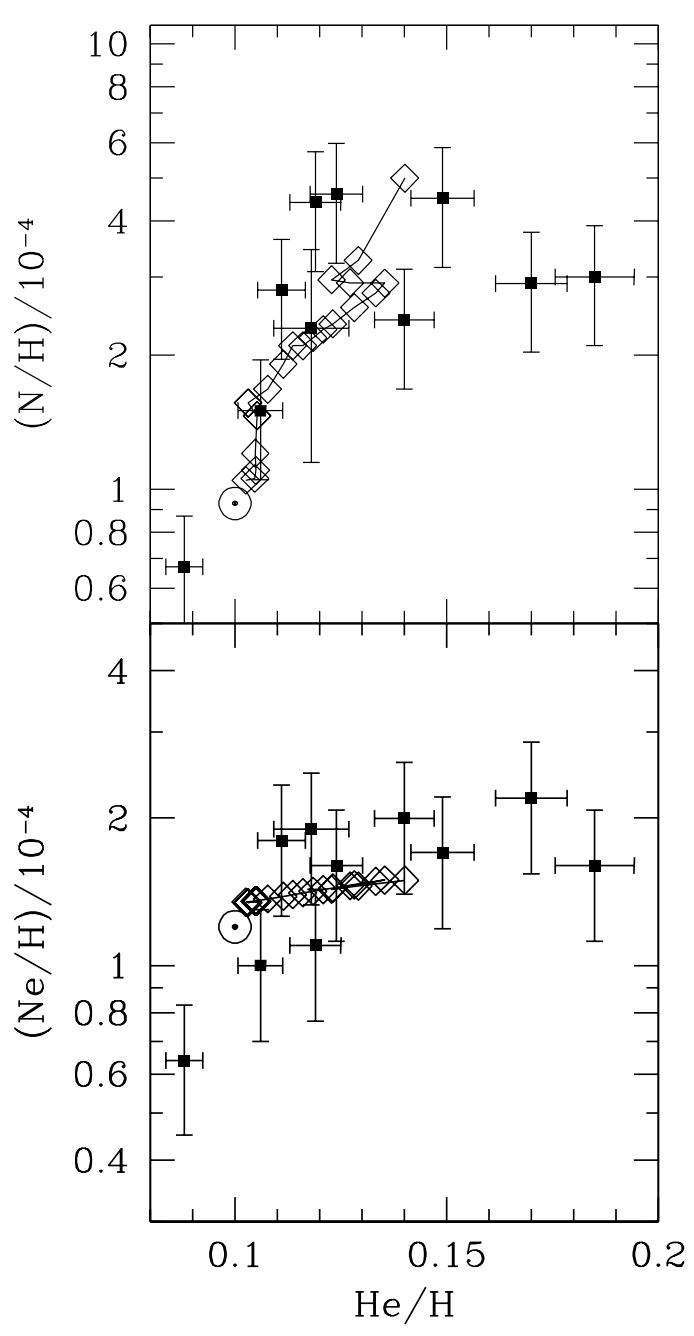

Fig. 3. Comparison between measured PN abundances (squares with errors bars) and model predictions (diamonds). Two estimates for the oxygen abundance for the Sun are also indicated, $\mathrm{O}_{\odot}$ High and $\mathrm{O}_{\odot}$ Low (see Table 4 and text). In each panel the sequence of diamonds denotes the expected PN abundance as a function of the stellar progenitor's mass, increasing from 0.9 to $5.0 M_{\odot}$ (a few values are labeled along $\mathrm{C} / \mathrm{H}$ curve), and for initial solar metallicity $Z=0.019$. The initial $\mathrm{O}$ abundance was set equal to $\mathrm{O}_{\odot}$ High. The model assumptions adopted for the TP-AGB evolution, corresponding to case A) of Table 4, include solar-scaled molecular opacities $\left(\kappa_{\mathrm{fix}}\right)$, and third dredge-up efficiency $\lambda=0.5$.
Henry et al. (2000) and Kingsburgh \& Barlow (1994) for PNe in the Milky Way, and from Dopita et al. (1997) for PNe in the LMC. Only PNe with measured C abundances and not already present in the ISO sample have been included. The reader should bear in mind that excluding helium, the errors on the remaining abundances of these two samples are large and uncertain.

The "normal" helium group in the ISO sample (with $\mathrm{He} / \mathrm{H}<0.145$; smaller filled squares) is within the range of abundances given by Henry et al. (2000) and Kingsburgh \& Barlow (1994). However, both studies lack PNe with high He abundances. Actually, PN PB 6 (from Henry et al. 2000 sample) has a helium abundance of 0.2 but that value is highly uncertain, and 0.146 is the highest helium abundance among those PNe that we have excluded from the Kingsburgh \& Barlow (1994) sample (those with no measured carbon).

Conversely, the three ISO PNe with high helium abundances (with $\mathrm{He} / \mathrm{H}>0.145$; larger filled squares) agree well with the LMC PNe from Dopita et al. (1997), not only in the $\mathrm{He}$ abundance but also in the $\mathrm{N} / \mathrm{O}$ and $\mathrm{C} / \mathrm{N}$ ratios. Besides supporting the division of the ISO sample into two subclasses, this also suggests that an initial sub-solar metallicity may be a key factor in the subsequent nucleosynthesis.

For all these reasons, it seems advantageous to separate those PNe which exhibit high helium content from the others having "normal" helium abundances, and to discuss the two groups separately.

\subsection{PNe with "normal" He abundances}

Let us examine the $\mathrm{PN}$ data with $\mathrm{He} / \mathrm{H} \lesssim 0.14$ (Fig. 3). For the sake of clarity we summarise the main features pointed out in Sect. 3.1.

- Most of the data present a clear enhancement in $\mathrm{He}, \mathrm{C}$, $\mathrm{N}$ compared to the abundances of these elements for the Sun. In particular, some of them should descend from carbon stars given their $\mathrm{C} / \mathrm{O}$ ratio larger than one.

- Oxygen abundances are underabundant when compared to the Solar determination by Anders \& Grevesse (1989). On the other hand they are consistent with a constant, or slightly increasing trend with $\mathrm{He} / \mathrm{H}$, if compared to the recent oxygen estimation for the Sun by Allende Prieto et al. (2001).

- Neon seems to exhibit a constant, or perhaps moderately increasing trend with $\mathrm{He} / \mathrm{H}$.

We first consider the elemental changes expected after the first and second dredge-up episodes. The predicted envelope abundances are displayed in Fig. 4 for two values of the initial metallicity, i.e. $Z=0.019$ and $Z=0.008$. Compared to their 


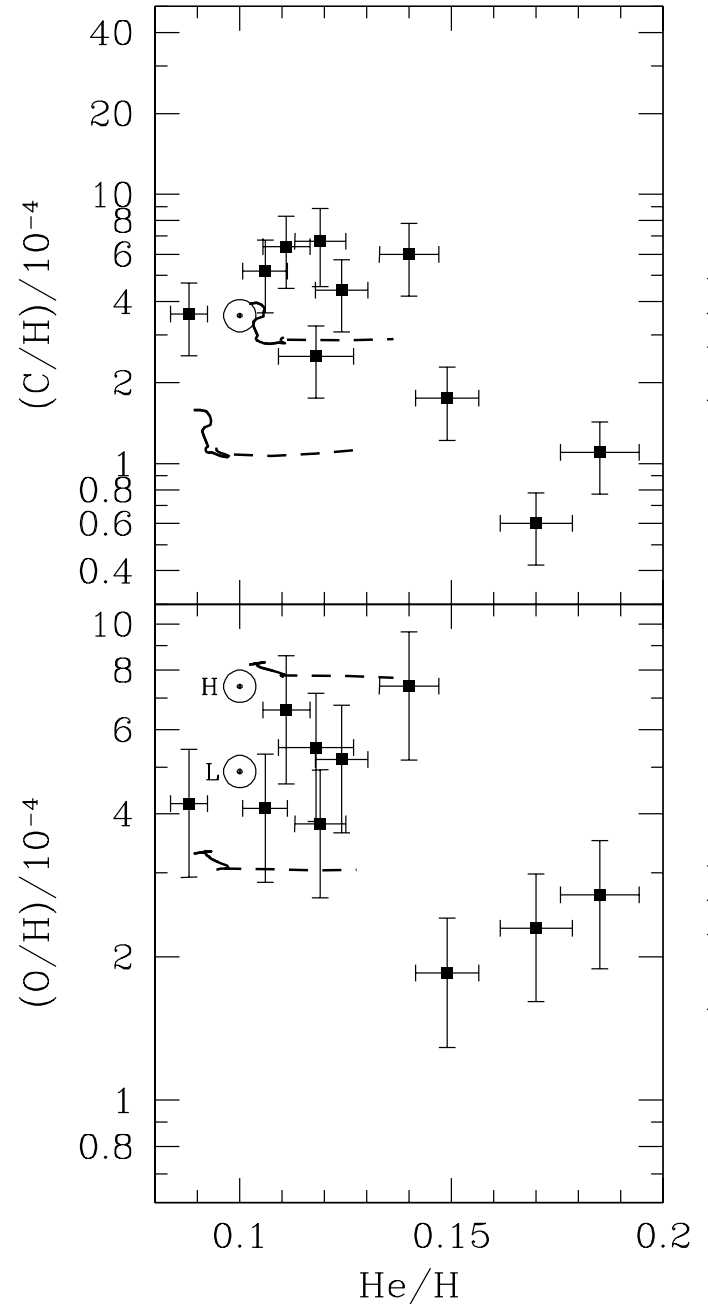

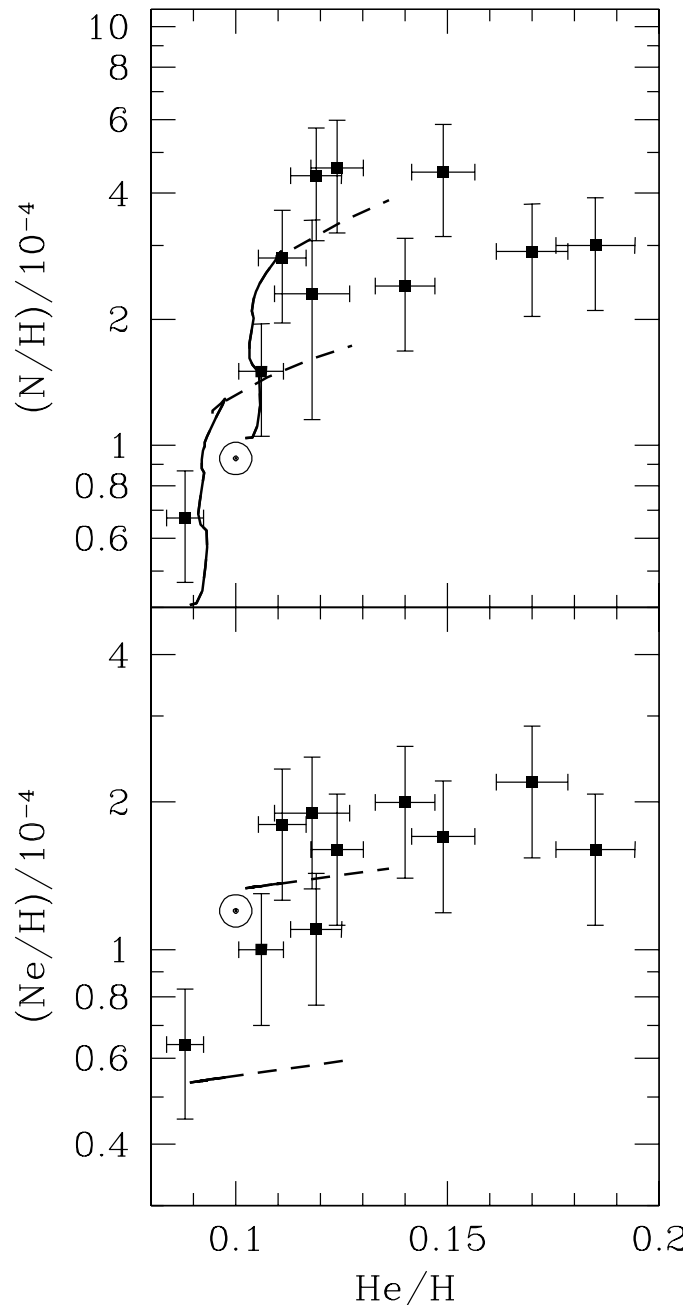

Fig. 4. Observed PN abundances (squares with error bars) compared to expected surface abundances (taken from Girardi et al. 2000) just before the onset of the TP-AGB phase, as a function of the initial stellar mass, and for two values of the original metallicity, i.e. $Z=0.019$ (upper curves) and $Z=0.008$ (lower curves). The initial mass ranges from 0.6 to $5.0 M_{\odot}$ at increasing $\mathrm{He} / \mathrm{H}$ along the curves. Predicted abundances are those expected after the first dredge-up (solid line) for stars with $0.6 \leq M / M_{\odot} \leq 4.0$; and after the second dredgeup (dashed line) for stars with $4.0<M / M_{\odot} \leq 5.0$. original values oxygen and neon are essentially unaffected, carbon is somewhat reduced, while nitrogen and helium may be significantly increased particularly after the second dredge-up in stars of intermediate mass $\left(M>4 M_{\odot}\right)$.

The comparison with the PN data indicates the necessity to invoke further chemical changes in addition to those caused by the first and second dredge-up, especially if one considers the observed enhancement in carbon. The most natural explanation resides in the third dredge-up process occurring during the TP-AGB phase. To this respect, as already mentioned in Sect. 6.1, the "old" models with fixed solar-scaled molecular opacities predict too large carbon enrichment (Fig. 3). This point and its possible solution are discussed below.

\subsubsection{Reproducing the extent of carbon enrichment}

To tackle the problem of carbon we consider a stellar model with $2 M_{\odot}$ initial mass, a typical mass of carbon stars in the Galactic disk (Groenewegen et al. 1995). Figure 6 shows a few models of the predicted carbon abundance and corresponding $\mathrm{PN}$ values.

As starting model we consider the one with the $\kappa_{\text {fix }}$ assumption. It is clearly located well above the observed points (diamond in left panel of Fig. 6).
A first significant improvement is obtained acting on the opacity, that is adopting variable molecular opacities during the TP-AGB calculations. We replace $\kappa_{\text {fix }}$ with $\kappa_{\mathrm{var}}$, while keeping the other model parameters fixed. In this model (starred symbol) the predicted $\mathrm{C} / \mathrm{H}$ is lowered because of a shorter $\mathrm{C}$-star phase, hence a decrease in the number of dredge-up episodes (see Marigo 2002, 2003).

Additional cases are explored. The usual assumption of constant dredge-up efficiency $\lambda$ is replaced with the recent recipe by Karakas et al. (2002), who provide analytic relations - fitting the results of full AGB calculations - that express the evolution of $\lambda$ as a function of metallicity $Z$, stellar mass $M$, and progressive pulse number. For a given $M$ and $Z, \lambda$ is found to increase from initially zero up to nearly constant maximum value, $\lambda_{\max }$. This latter also varies depending on mass loss. Adopting $\lambda_{\max }=0.457$ as suggested by Karakas et al. (2002) for the $\left(M=2 M_{\odot}, Z=0.02\right)$ combination, we end up with a somewhat lower $\mathrm{C} / \mathrm{H}$ (triangle), compared to the case with $\left(\kappa_{\mathrm{var}}\right.$, $\lambda=0.5)$. This reflects the smaller amount of carbon globally dredged-up with the $\lambda_{\text {var }}$ assumption.

A further test is made with respect to the assumed initial oxygen abundance. We calculate the TP-AGB evolution of the $2 M_{\odot}$ model replacing the surface oxygen abundance at the first thermal pulse - as predicted by Girardi et al. (2000) evolutionary calculations, based on Anders \& Grevesse (1989) 

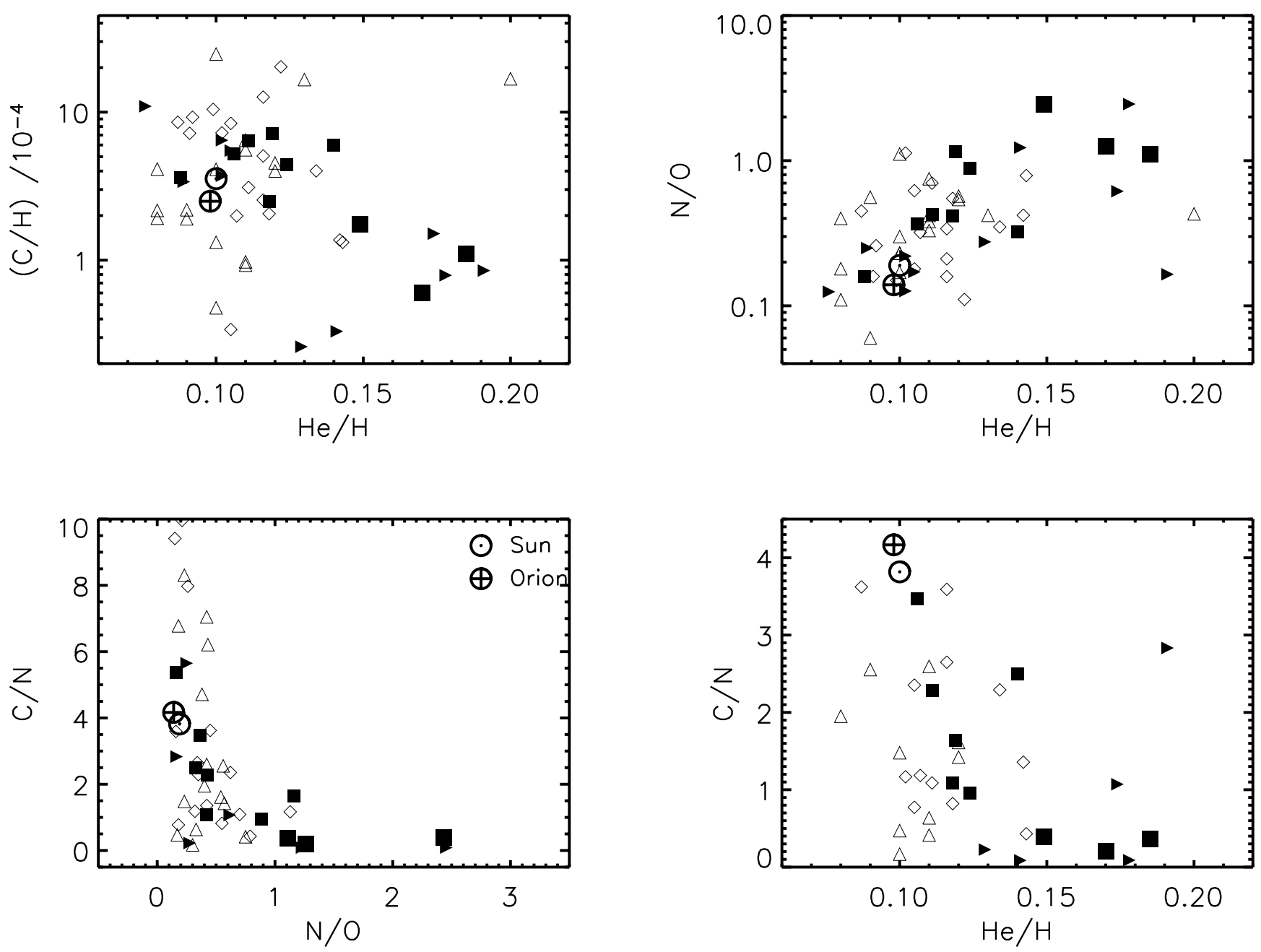

Fig. 5. Comparison of PN abundances from our ISO sample (filled squares) and other samples in the literature, namely: Henry et al. (2000; open triangles) and Kingsburgh \& Barlow (1994; diamonds) for PNe in the Milky Way, and Dopita et al. (1997; filled triangles) for PNe in the LMC. The ISO sample has been plotted using smaller and larger squares for PNe with helium abundances lower and higher than 0.145 respectively.

solar mixture (the $\mathrm{O}_{\odot}$ High in Table 4 ) - by the recently revised determination by Allende Prieto et al. (2001; the $\mathrm{O}_{\odot}$ Low in Table 4).

With the new lower oxygen abundance the solar $\mathrm{C} / \mathrm{O}$ ratio increases from 0.48 to 0.78 . This increment in the initial $\mathrm{C} / \mathrm{O}$ ratio has important effects on the later TP-AGB evolution and PN abundances: fewer dredge-up episodes are necessary to produce a carbon star and hence, on average, a lower $\mathrm{C}$ abundance is expected in the PN ejecta. This can be seen in Fig. 6, by comparing the models labeled by triangle and circle symbols. We also notice that the trend in the predicted values of $\mathrm{C} / \mathrm{H}$ (left panel) or $\mathrm{C} / \mathrm{O}$ (right panel) is reversed. The $\mathrm{O}_{\odot}$ Low prescription leads to a reduced absolute carbon enrichment compared to the $\mathrm{O}_{\odot}$ High case, while the final $\mathrm{C} / \mathrm{O}$ ratio is larger in the former case.

At this point the results already appear better compared to the starting model, but in order to carry the expected $\mathrm{C} / \mathrm{H}$ point within the observational error bars, we change another fundamental model parameter, i.e. the dredge-up efficiency $\lambda$. A good fit of the PNe data is obtained by lowering $\lambda_{\max }$ from 0.457 to 0.3 , that simply means diminishing the amount of dredgedup carbon.

In summary, from these calculations we derive the following indications. Both the $\mathrm{C} / \mathrm{H}$ and the $\mathrm{C} / \mathrm{O}$ ratios of the carbonrich Galactic PNe - evolved from stars with typical masses of $\sim 2.0 M_{\odot}-$ can be reproduced by assuming i) variable molecular opacities $\kappa_{\mathrm{var}}$, ii) an initial oxygen abundance as recently revised by Allende Prieto et al. (2001), and iii) a dredge-up efficiency $\lambda \approx 0.3-0.4$. These indications - in particular points i) and iii) - agree with the results recently obtained by Marigo $(2002,2003)$ in her analysis of the properties of Galactic carbon stars in the disk. Variable molecular opacities and $\lambda \lesssim 0.5$ are required to reproduce a number of observables of carbon stars, like their $\mathrm{C} / \mathrm{O}$ ratios, effective temperatures, mass-loss rates, and near-infrared colours.

Then, from the representative case of the $2.0 M_{\odot}$ model, we consider a wider mass range, i.e. $1.1-5.0 M_{\odot}$, with initial metallicity $Z=0.019$. Results of the $\mathrm{C} / \mathrm{H}$ and $\mathrm{C} / \mathrm{O}$ ratios as a function of $\mathrm{He} / \mathrm{H}$, expected in the corresponding PNe, are displayed in Figs. 7 and 8 and are also summarized in the topleft panels of Figs. 11 and 12 (triangles connected by solid 

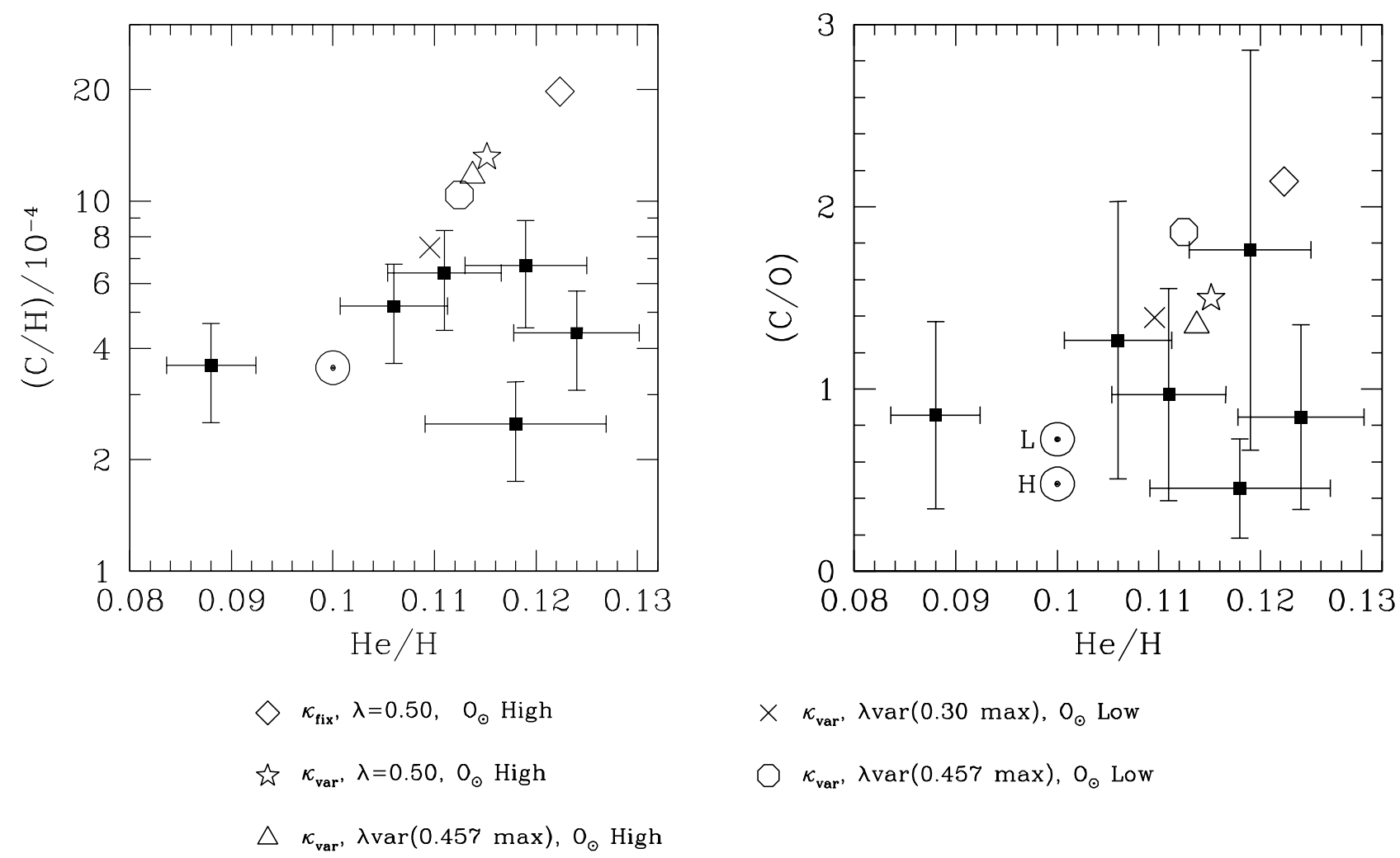

$\times \kappa_{\text {var }}, \lambda \operatorname{var}(0.30 \max ), O_{\odot}$ Low

$\kappa_{\mathrm{var}}, \lambda \operatorname{var}(0.457 \max ), \mathrm{O}_{\odot}$ Low

Fig. 6. Carbon abundances and $\mathrm{C} / \mathrm{O}$ ratios in $\mathrm{PNe}$ with low helium abundances $(\mathrm{He} / \mathrm{H} \leq 0.13)$. Open symbols denote predictions of synthetic TP-AGB calculations for various choices of model parameters - specified in the legenda - corresponding to a stellar progenitor with $\left(M_{\mathrm{i}}=\right.$ $\left.2.0 M_{\odot}, Z_{\mathrm{i}}=0.019\right)$. See text and cases B-C-D-E of Table 4 for more details.

line). They show a satisfactory agreement with the observed data for $\mathrm{He} / \mathrm{H}<0.14$, suggesting a mass interval for the stellar progenitors from about 1.0 to $4.0 M_{\odot}$.

Synthetic TP-AGB calculations are carried out by adopting the $\kappa_{\mathrm{var}}$ and $\mathrm{O}_{\odot}$ Low prescriptions, and assuming $\lambda \approx 0.3-0.5$ for lower stellar masses $\left(M \lesssim 3.0 M_{\odot}\right)$, while increasing it up to $\lambda \approx 0.98$ for the largest masses. This means that the third dredge-up should become deeper in more massive stars, as indicated by full TP-AGB calculations (e.g. Vassiliadis \& Wood 1993; Karakas et al. 2002). It is worth noticing that models with $M \leq 1.5 M_{\odot}$ are predicted not to experience the third dredge-up, i.e. they do not fulfil the adopted temperature criterion (based on the parameter $T_{\mathrm{b}}^{\text {dred; }}$; refer to Sect. 5.1).

Moreover, we assume that in the most massive stars (with $M=4.0-5.0 M_{\odot}$ ) the composition of the inter-shell may be different from the standard one, i.e. less primary carbon is supposed to be synthesised during thermal pulses. This point will be discussed in more detail in Sect. 6.4.2.

\subsubsection{Other elemental abundances}

As for the $\mathrm{He}, \mathrm{N}, \mathrm{O}$, Ne elemental abundances, we can outline the following (see sequences of triangles in Figs. 11 and 12):

- The helium abundance up to $\mathrm{He} / \mathrm{H} \sim 0.14$ is well reproduced by accounting for the surface enrichment due to the first, and possibly second and third dredge-up. Apparently, for this group of $\mathrm{PNe}$, there is no need to invoke a significant production of helium by HBB. We also notice that the minimum value predicted by stellar models with initial solar composition is $\mathrm{He} / \mathrm{H} \sim 0.096$, so that any observed value lower than that (e.g. NGC 7662) may correspond to a stellar progenitor with lower initial metallicity and helium content.

- The nitrogen data are consistent, on average, with the expected enrichment produced by the first and second dredgeup events.

- As already discussed, the PN oxygen estimates are compatible with the recent revision for the abundance in the Sun by Allende Prieto et al. $\left(2001 ;\left(\mathrm{O} / \mathrm{H}_{\odot}\right)=4.9 \times 10^{-4}\right)$. The PN data indicate that during the evolution of the stellar progenitors, their surface abundance of oxygen is essentially unchanged, or it might be somewhat enhanced. Anyhow, the revised lower determination for the Sun removes the problem of explaining the systematic oxygen under-abundance compared to solar that all data would present if adopting higher values for the Sun as indicated by past analyses (e.g. Anders \& Grevesse 1989; $(\mathrm{O} / \mathrm{H})_{\odot}=$ $\left.7.4 \times 10^{-4}\right)$.

- The neon data do not allow to put stringent constraints on the nucleosynthesis of this element in TP-AGB stars with initial solar metallicity. In fact, within the uncertainties the observed $\mathrm{Ne} / \mathrm{H}$ abundances are compatible with a preservation of the original value, but they can also point to a moderate increase. 


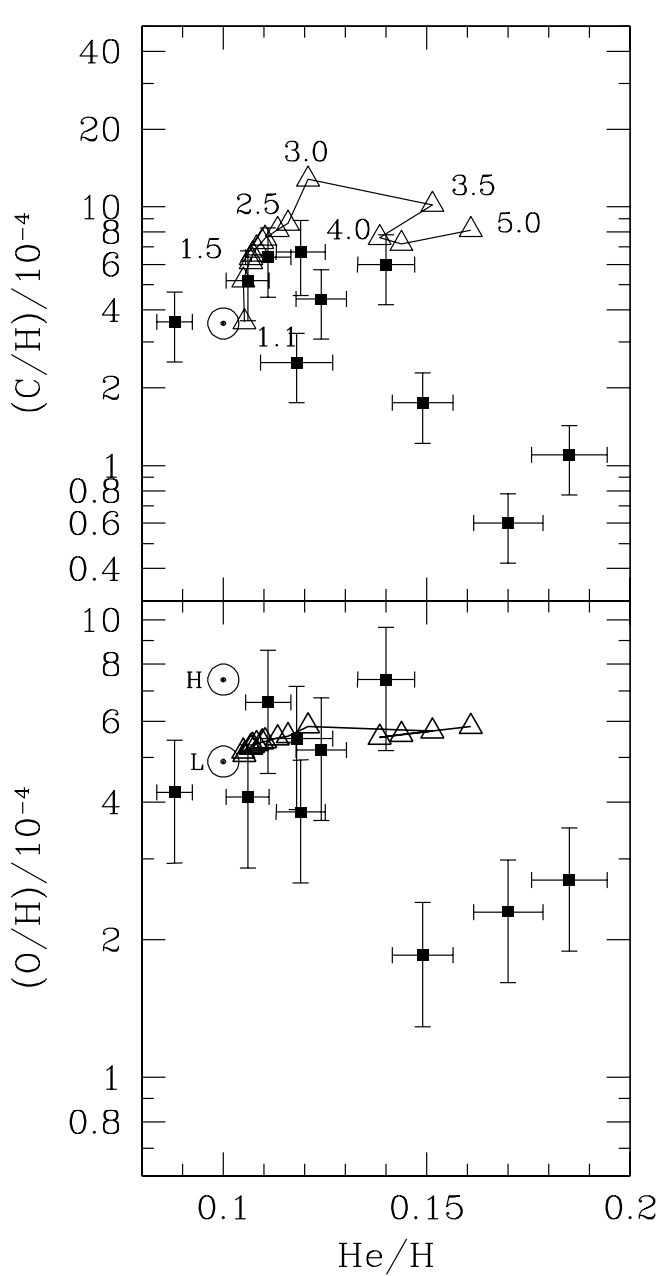

Predictions shown in Fig. 11 are obtained under the assumption that the synthesis of ${ }^{22} \mathrm{Ne}$ - via $\alpha$-captures in the He-burning shell starting from ${ }^{14} \mathrm{~N}$ during thermal pulses - takes place with almost maximum possible efficiency $(F=0.99$, see Sect. 5.1). As we see, the final expected enrichment in PNe remains modest, due to the relatively small number of thermal pulses and moderate dredge-up efficiency $(\lambda \sim 0.3-0.5)$ characterising TP-AGB models with initial masses $M \sim 1.5-3.0$, and metallicity $Z=0.019$.

The opposite situation, that is the complete conversion of ${ }^{22} \mathrm{Ne}$ into ${ }^{25} \mathrm{Mg}$ with $F \sim 0$, would not lead to any enrichment in neon so that the sequence of predicted $\mathrm{Ne} / \mathrm{H}$ would be flatter ${ }^{2}$ than the present one (connected triangles in Fig. 11). But this case could not be rejected either since corresponding predictions are still confined within the observed domain.

\subsection{PNe with extremely high He abundance}

Below we discuss the PNe exhibiting the largest enrichment in helium, i.e. with $0.14 \lesssim \mathrm{He} / \mathrm{H} \lesssim 0.20$ (see e.g. Fig. 3). These

\footnotetext{
${ }^{2}$ In any case $\mathrm{Ne} / \mathrm{H}$ should keep a slightly increasing trend, not becoming exactly horizontal since, even if $\mathrm{Ne}=$ const., the hydrogen content $\mathrm{H}$ decreases as a consequence of dredge-up events.
}

objects share other chemical properties, namely:

- marked carbon deficiency compared to the solar value;

- sizeable enhancement of nitrogen, but not exceeding the upper values observed in the $\mathrm{PNe}$ with lower $\mathrm{He} / \mathrm{H}$;

- significant depletion of oxygen, which makes these PNe appear as a distinct group with respect to the PNe with lower $\mathrm{He} / \mathrm{H}$ abundances;

- possible, but still not compelling, hint of over-abundance of neon compared to the solar value.

By looking at Fig. 4, we conclude that model predictions after the first and second dredge-up processes cannot account for the extremely high $\mathrm{He} / \mathrm{H}$ values of these PNe. Then, we are led to consider additional He contributions from the third dredge-up and HBB during the TP-AGB phase.

Qualitatively, the enrichment in helium and nitrogen and the simultaneous deficiency in carbon would naturally point to $\mathrm{HBB}$ as a possible responsible process, via the CNO-cycle reactions. Therefore, as a working hypothesis, let us assume that the stellar progenitors of these extremely He-rich PNe are intermediate-mass stars $\left(M \gtrsim 4.5 M_{\odot}\right)$, experiencing HBB during their AGB evolution. We will now investigate under which conditions all elemental features can be reproduced.

To allow an easier understanding of the following analysis, Figs. 9 and 10 show the predicted time evolution of $\mathrm{He}$, $\mathrm{C}, \mathrm{N}, \mathrm{O}$, and $\mathrm{Ne}$ elemental abundances in the envelope during 

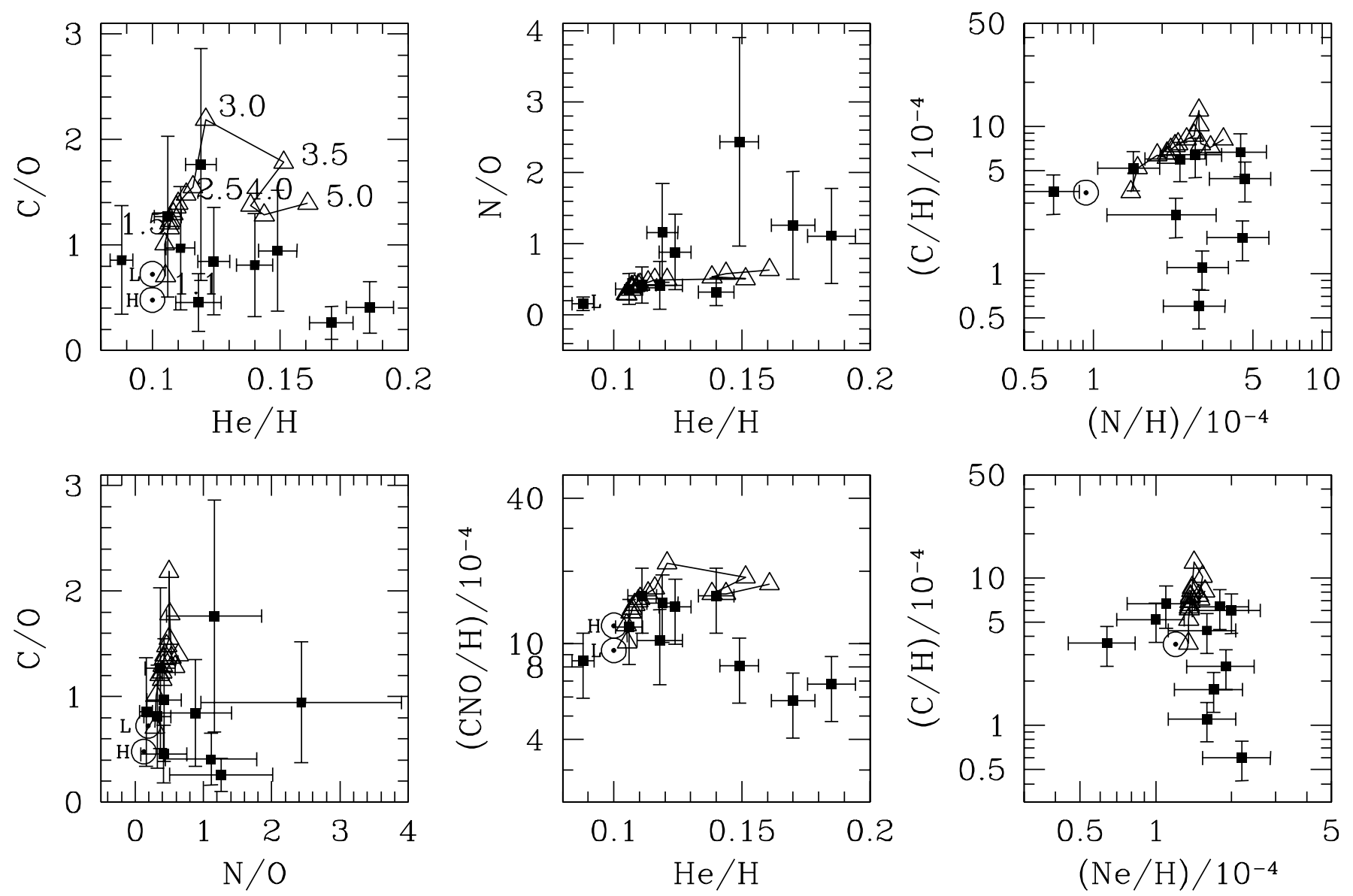

Fig. 8. The same as in Fig. 7, but expressing the abundance data with different combinations of elemental ratios.

the TP-AGB phase of models experiencing HBB. Different assumptions are explored. The observed PN abundances should be compared with the last starred point along the theoretical curves, which marks the last event of mass ejection, and it may be then considered representative of the expected PN abundances.

\subsubsection{Constraints from oxygen and sulfur abundances}

At this point additional information comes from the marked oxygen under-abundance compared to solar (for both High and Low values), common to the extremely He-rich PNe.

We recall that the oxygen abundance in the envelope remains essentially unchanged after the first and second dredgeup events. The third dredge-up may potentially increase oxygen, depending on the chemical composition of the convective inter-shell that forms at thermal pulses. In any case, no oxygen depletion is expected by any of these processes. A destruction of oxygen could be caused by a very efficient HBB, that is if the ON cycle is activated and oxygen starts being transformed into nitrogen.

We have explored this possibility on a $5 M_{\odot}$ TP-AGB model with original solar metallicity. To analyse the effects of a larger HBB efficiency, the mixing-length parameter $\alpha_{\mathrm{ML}}$ has been increased, and set equal to 1.68, 2.00, and 2.50. In fact, larger values of $\alpha_{\mathrm{ML}}$ correspond to higher temperatures at the base of the convective envelope. In none of the three cases have we found any hint of oxygen destruction, as indicated by the flat behaviour of the abundance curves in the bottom-left panel of Fig. 9 (solid and long-dashed lines, for $\alpha=1.68$ and 2.50, respectively).

At this point we decided to stop further increasing $\alpha-$ which would have likely led to oxygen destruction at some point - since we run into a major discrepancy. In fact, increasing the efficiency of HBB causes a systematic over-enrichment in nitrogen, as shown by the model with $\alpha_{\mathrm{ML}}=2.50$ (shortdashed line). We also note that a significant nitrogen production is accompanied by a mirror-like destruction of carbon (upperleft panel of Fig. 9). This is not the case of the model with $\alpha_{\mathrm{ML}}=1.68$ (solid line), in which HBB is almost inoperative.

From these results we can expect that, even if a destruction of oxygen is obtained for larger values of $\alpha_{\mathrm{ML}}$, the problem of nitrogen over-production would become even more severe. All these considerations suggest that the most He-rich PNe in the sample should not descend from stars with original solar metallicity, but rather from more metal-poor progenitors. In other words, the observed sub-solar oxygen abundances likely reflect the initial stellar metallicity.

To test the hypothesis of an original lower oxygen content we perform explorative TP-AGB evolutionary calculations of intermediate-mass stars with initial LMC composition, characterised by roughly half-solar metallicity. The predicted envelope abundances after the first and second dredge-up for stellar 

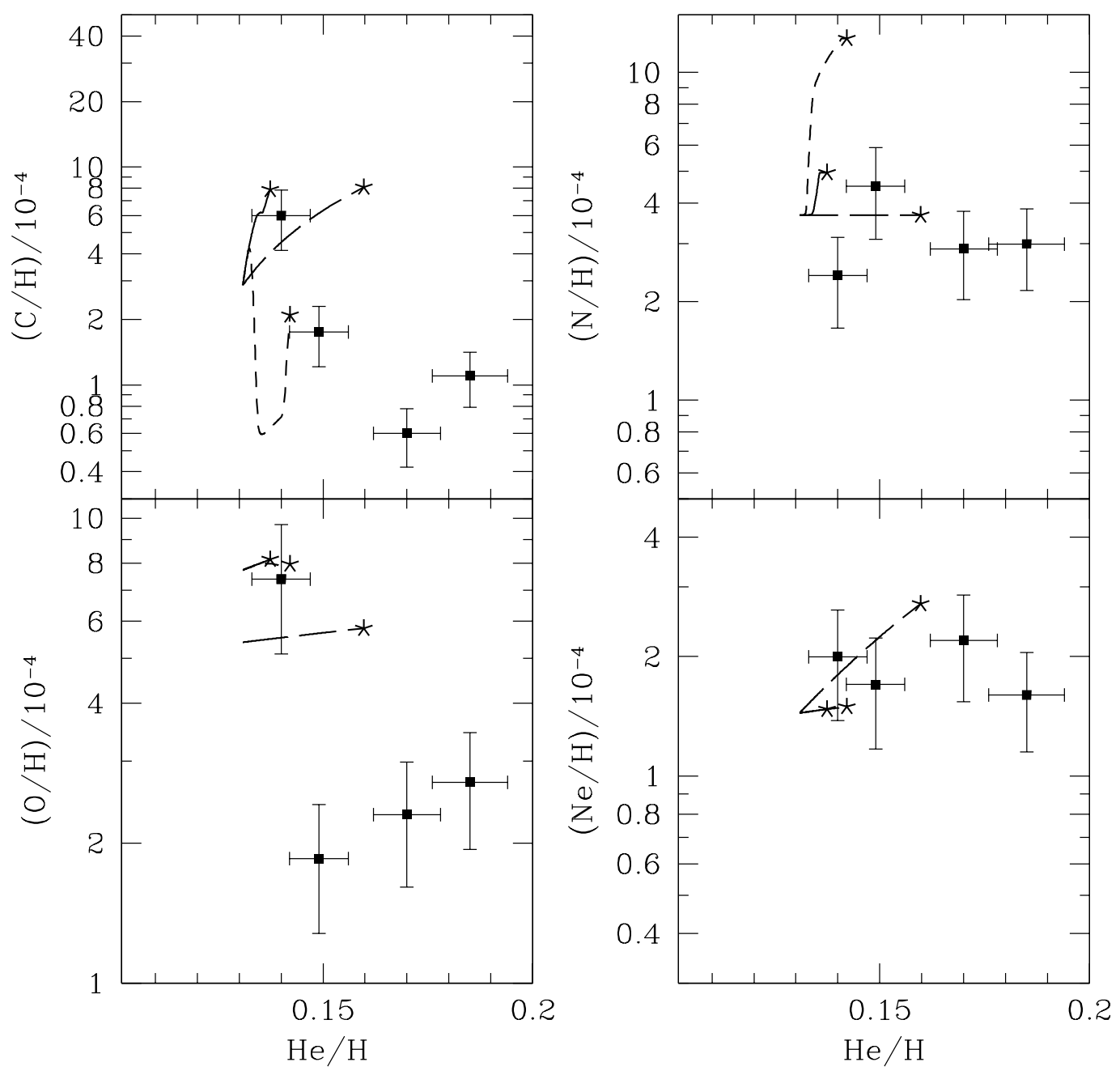

Fig. 9. Time evolution of surface elemental abundances during the TP-AGB phase of a $5.0 M_{\odot}$ model with initial solar chemical composition, experiencing both the third dredge-up and HBB. Observed PN data should be compared with the starred symbol at the end of each curve (marking the end of the TP-AGB phase). Most parameter prescriptions are specified Table 4. In practice we consider the following cases: i) efficient $\mathrm{HBB}$ with $\alpha=$ 2.50 (short-dashed line; refer to case A) of Table 4 for other model parameters); ii) weak HBB with $\alpha=1.68$ (solid line; refer to case A) of Table 4); and iii) weak HBB and efficient dredge-up, starting from a lower oxygen abundance (long-dashed line; refer to case F) of Table 4).

models with $[Z=0.008, Y=0.25]$ are shown in Fig. 4. As we see the oxygen curve (bottom-left panel) is now lower than the corresponding one for solar composition, and it appears to be much more consistent, but not fully, with the location of the He-rich PNe. In the next Sects. 6.4.2 and 6.4.3, we will test whether these models are actually able to fulfil, besides the oxygen data, all other chemical constraints related to $\mathrm{He}, \mathrm{C}$, $\mathrm{N}$, and $\mathrm{Ne}$ abundances. Finally, we note that even better results for $\mathrm{O}$ may be obtained by adopting an initial oxygen abundance for the LMC composition, scaled from the new solar determination by Allende Prieto et al. (2001).

It is worth adding now some considerations about the possibility that the most He-rich $\mathrm{PNe}$ in the sample evolved from stars with sub-solar metallicity.

On the one side, our proposed scenario may run into trouble if we suppose that the history of chemical enrichment of our Galactic disk simply follows an age-metallicity relation, in which younger ages correspond to larger metallicities. In fact in this case, we would expect that stars with initial masses as large as $\sim 5 M_{\odot}$ should form from gas with comparable, or even higher, degree of metal enrichment than the Sun. However, more detailed considerations show that the assumption of a unique age-metallicity relation provides an over-simplified description of the actual chemical evolution in the Galactic disk.
For instance, the Orion nebula has a lower metallicity than solar even though it is younger. Also, in the solar neighborhood differences do exist. Edvardsson et al. (1993) studied a sample of nearly $200 \mathrm{~F}$ and $\mathrm{G}$ dwarfs in the Galactic disk and found a considerable $[\mathrm{Fe} / \mathrm{H}]$ scatter even for stars with similar age and belonging to a nearby field of the disk. They concluded from this that the chemical enrichment in the Galaxy is inhomogeneous. There is therefore a fraction of disk stars in the sky with different composition from solar. The three PNe with high $\mathrm{He}$ abundance belong to the disk and are indeed in the same part of the sky (within $\pm 10^{\circ}$ of the Galactic center). We may reasonably state that these objects belong to lowest tail of the metallicity distribution in the Galactic disk. These arguments support the use of a metallicity different from solar but do not point to LMC metallicity. Another weak point is that the metallicity generally increases towards the center of the Galaxy, while these PNe should have a lower metallicity.

An additional hint for a different initial composition from solar is given by sulfur. All PNe in the sample display a clear under-abundance of sulfur with respect to solar (see Fig. 2), whereas this element is expected not to change in the course of stellar evolution. Its abundance in our PN sample is even lower than in Orion (see Table 3), which is a young stellar object. This implies that either the nucleosynthesis history of sulfur is not 

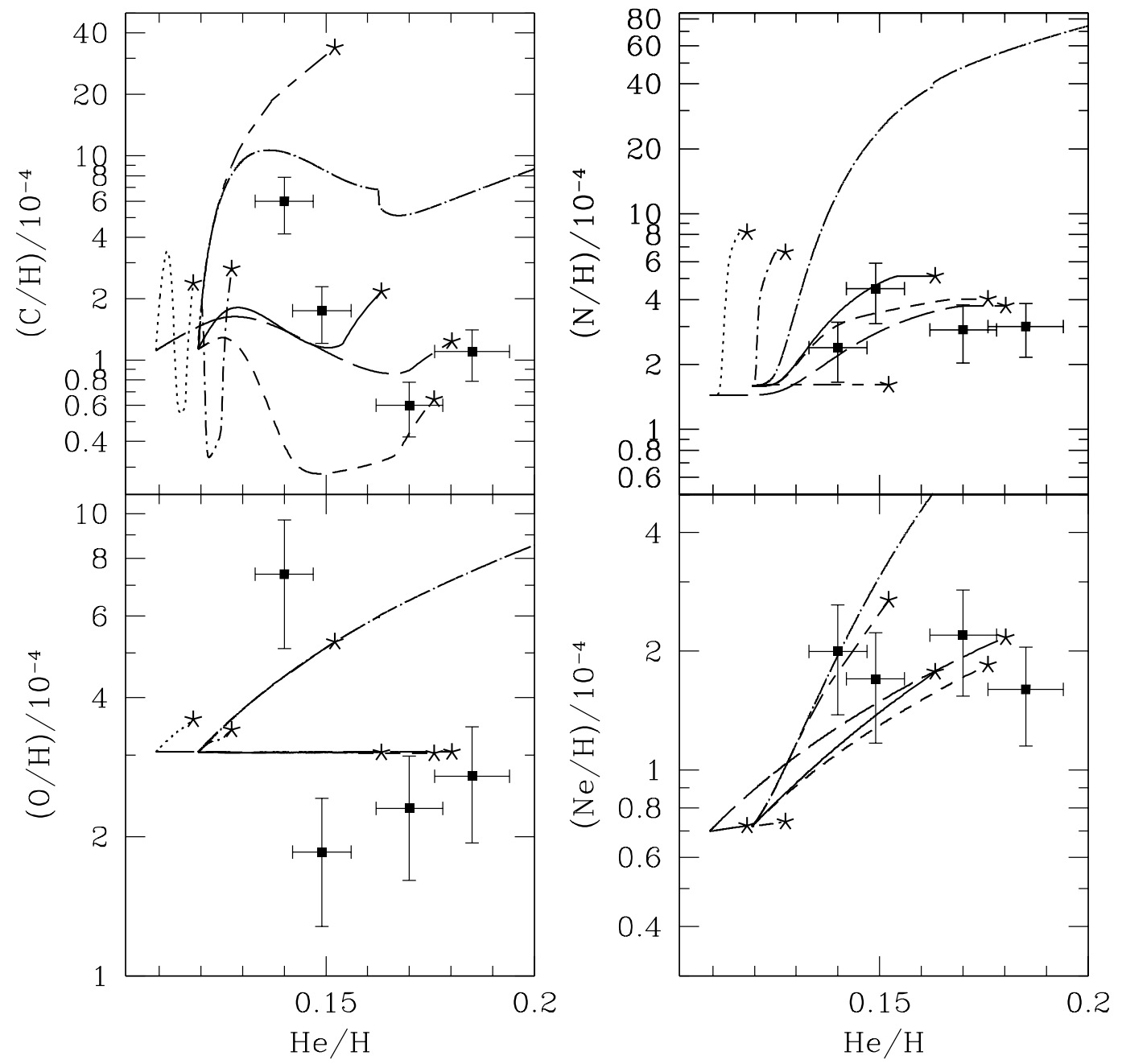

Fig. 10. The same as in Fig. 9, but for initial LMC chemical composition. Illustrated cases mainly differ in the adopted parameters describing the third dredge-up, i.e. (see also Table 4): i) and ii) moderate third dredge-up $(\lambda=0.5)$ and standard chemical composition of the inter-shell (case G) for 4.5 and $5.0 M_{\odot}$ models (dotted and dot-short-dashed lines, respectively), iii) deep third dredge-up $(\lambda=0.9)$ and standard chemical composition of the inter-shell (case $\mathrm{H}$ ) for $5.0 M_{\odot}$ model (dot-longdashed line); iv) the same as the previous case but for the $\kappa_{\mathrm{var}}$ prescription (case I; short-long-dashed line); v), vi), and vii) deep third dredge-up $(\lambda=0.9)$ and intershell chemical composition with low-carbon abundance for $5.0 M_{\odot}$ model (solid line and case $\mathrm{J}$; short-dashed line and case $\mathrm{K}$ ) and $4.5 M_{\odot}$ model (long-dashed line and case $\mathrm{K}$ ).

yet understood and it can actually be destroyed in the course of the evolution, or that the initial composition, at least of this element, can deviate from solar. Alternatively, the determination of the solar abundance may be wrong. This possibility seems the most plausible. The sulfur abundance is within the range of values given by Martín-Hernández et al. (2002) for a sample of galactic H II regions. If the initial abundance of sulfur is not solar, this may also be the case for the other elements. Maybe the He-rich PNe are consistent with sub-solar metallicities but, since all observed PNe are under-abundant in sulfur, we are left to explain why for some PNe we need solar metallicity whereas for others a lower metallicity is invoked, this latter feature only applying to the most He-rich objects. This might be the result of some, still unidentified, selection effect.

The PNe with high helium abundance also show the lowest $(\mathrm{C}+\mathrm{N}+\mathrm{O}) / \mathrm{H}$ abundance (see Fig. 2). This, again, suggests a lower initial metallicity. Nevertheless the neon, sulfur and argon abundances are similar to the rest of planetaries which would in principle be against this suggestion. The similarity in neon of these three PNe with the rest can be explained invoking the production of neon in the course of evolution (see Sect. 6.4.3). The LMC sulfur abundance is compatible with all the PNe in the sample. As discussed above it might be that the sulfur solar abundance is wrong since this is a primary element and the observed abundances are quite accurate. The argon is more tricky. All PNe show similar argon abundances which are actually close to solar. The LMC argon abundance is much lower than that of the high helium PNe. The discrepancy between the subsolar sulfur and the solar argon abundance is not readily explained.

These aspects still need to be clarified, but we would remark that a possible answer may come in the context of a dedicated study of the chemical evolution of our Galaxy, which is beyond the scope of the present work.

\subsubsection{Constraints from carbon and nitrogen abundances}

Perusal of the $\mathrm{C} / \mathrm{H}$ and $\mathrm{N} / \mathrm{H}$ curves in Fig. 4 (predicted after the first and second dredge-up) for the LMC composition, shows that they share the trends with the stellar mass as the solarmetallicity curves, but the carbon and nitrogen abundances are now systematically lower, as for oxygen. In particular carbon abundances, prior to the TP-AGB evolution, - at any stellar mass - are now compatible with the low values measured in the He-rich PNe. As for nitrogen, some enrichment seems instead 


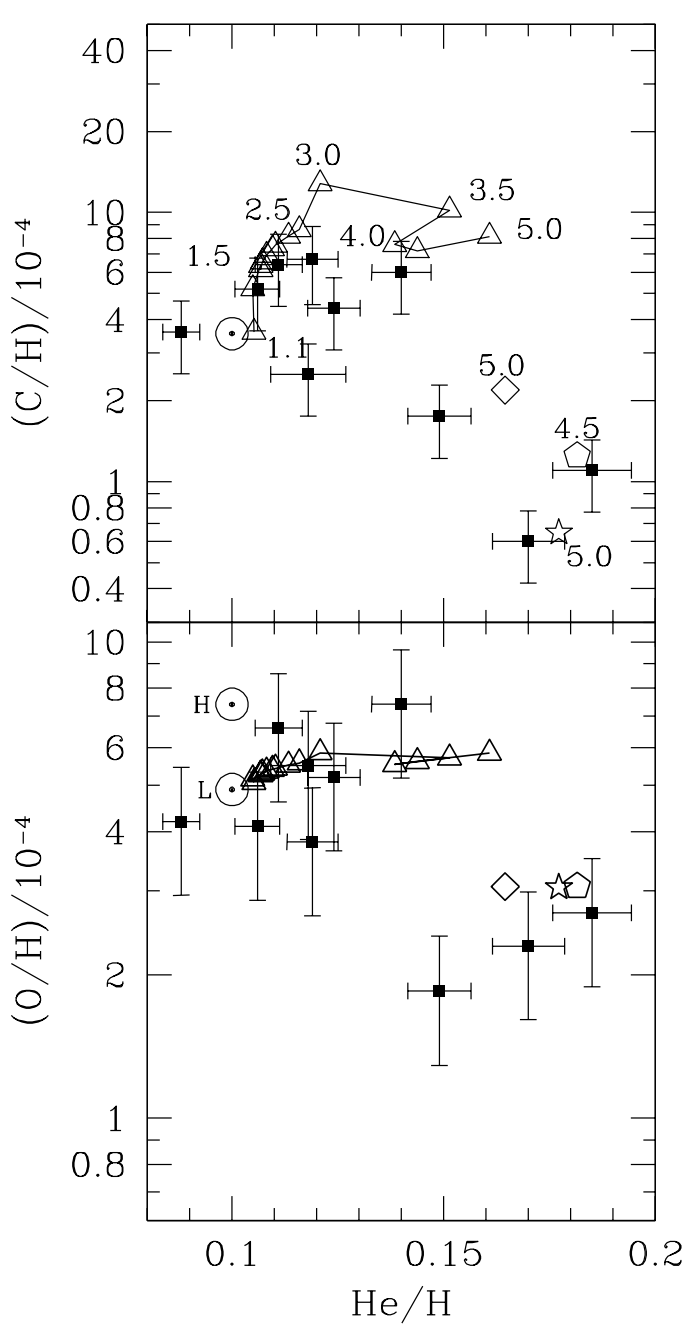

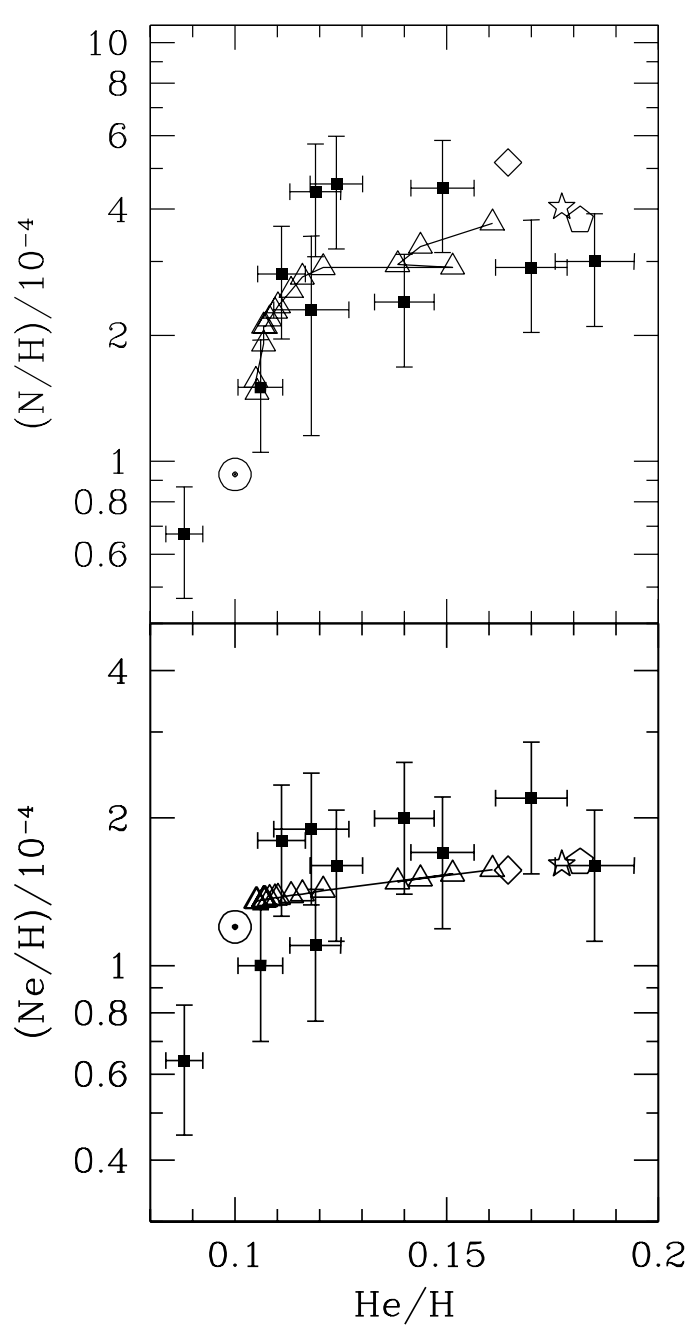

Fig. 11. Summary of the results that best fit the observed PN abundances. Specifically, triangles represent the same predictions as in Fig. 7, that are derived from TP-AGB models with $Z=0.019$ and with initial stellar masses from 1.1 to $5.0 M_{\odot}$. The other three symbols correspond to intermediate-mass stars with initial LMC composition $(Z=$ $0.008)$, namely: pentagon for the $4.5 M_{\odot}$ model with K) prescriptions, star for the $5.0 M_{\odot}$ model with $\mathrm{K}$ ) prescriptions, and diamond for the $5.0 M_{\odot}$ model with J) prescriptions. See text for more details. required (up to a factor of 2 at the largest stellar masses) to obtain agreement with the observations.

Given these premises, a number of TP-AGB models with $Z=0.008$ and masses of 4.0-5.0 $M_{\odot}$ have been calculated for different choices of parameters, in view to simultaneously matching the observed carbon, nitrogen and helium abundances. We just summarise the main emerging points.

Efficiency of the third dredge-up. High He abundances strongly depend on the efficiency of the third dredge-up. Figure 10 shows that relatively low values of $\lambda$ lead to inadequate helium enrichment, because both a limited amount of helium is dredged-up at each thermal pulse, and the duration of the TP-AGB phase becomes shorter, implying fewer dredge-up events. This is true for the TP-AGB models with $M=4.5,5.0 M_{\odot}$, and $\lambda=0.5$ (dotted and dot-short-dashed curves), which do not go beyond $\mathrm{He} / \mathrm{H}=0.13$.

On the other hand, TP-AGB models of the same initial masses but with $\lambda \sim 0.9$ become highly enriched in helium, yielding a very close agreement with the observed data for $\mathrm{He} / \mathrm{H}$ (solid, short-dashed, and long-dashed curves). The largest $\mathrm{He} / \mathrm{H}$ values measured in $\mathrm{PNe}$ are reproduced under the assumption that massive TP-AGB stars experience, besides HBB, a large number (of the order of 100) of very efficient third dredge-up episodes.

This indication is supported by recent results of full TP-AGB calculations for stellar masses $\gtrsim 5 M_{\odot}$ (Vassiliadis \& Wood 1993; Frost et al. 1998; Karakas et al. 2002).

The problem of nitrogen over-production. Invoking a large dredge-up efficiency does not guarantee, however, that all other elemental features are reproduced. In fact, assuming a very efficient dredge-up $\lambda \sim 0.9$, and the standard intershell chemical composition (i.e. $X_{\mathrm{csh}}\left({ }^{12} \mathrm{C}\right)=0.22, X_{\mathrm{csh}}\left({ }^{16} \mathrm{O}\right)=0.02$, $X_{\text {csh }}\left({ }^{4} \mathrm{He}\right)=0.76$; see Sect. 5.1), we find a sizeable overproduction of nitrogen by HBB. This is illustrated in Fig. 10 by the $\mathrm{N} / \mathrm{H}$ curve corresponding to the $5 M_{\odot}$ model (with $\kappa_{\text {fix }}$ prescription; dot-long-dashed line). Calculations were stopped before the termination of the TP-AGB evolution (i.e. before the ejection of the entire envelope), since the $\mathrm{He} / \mathrm{H}$ in the envelope already exceeded 0.2 and the over-production of nitrogen was very large.

In relation to this latter point, going back to the past literature, the same problem was already pointed out by Marigo et al. (1998; see their Sect. 5.4) and earlier by Becker \& Iben (1980; see their Sect. VII), in their careful analysis on the 

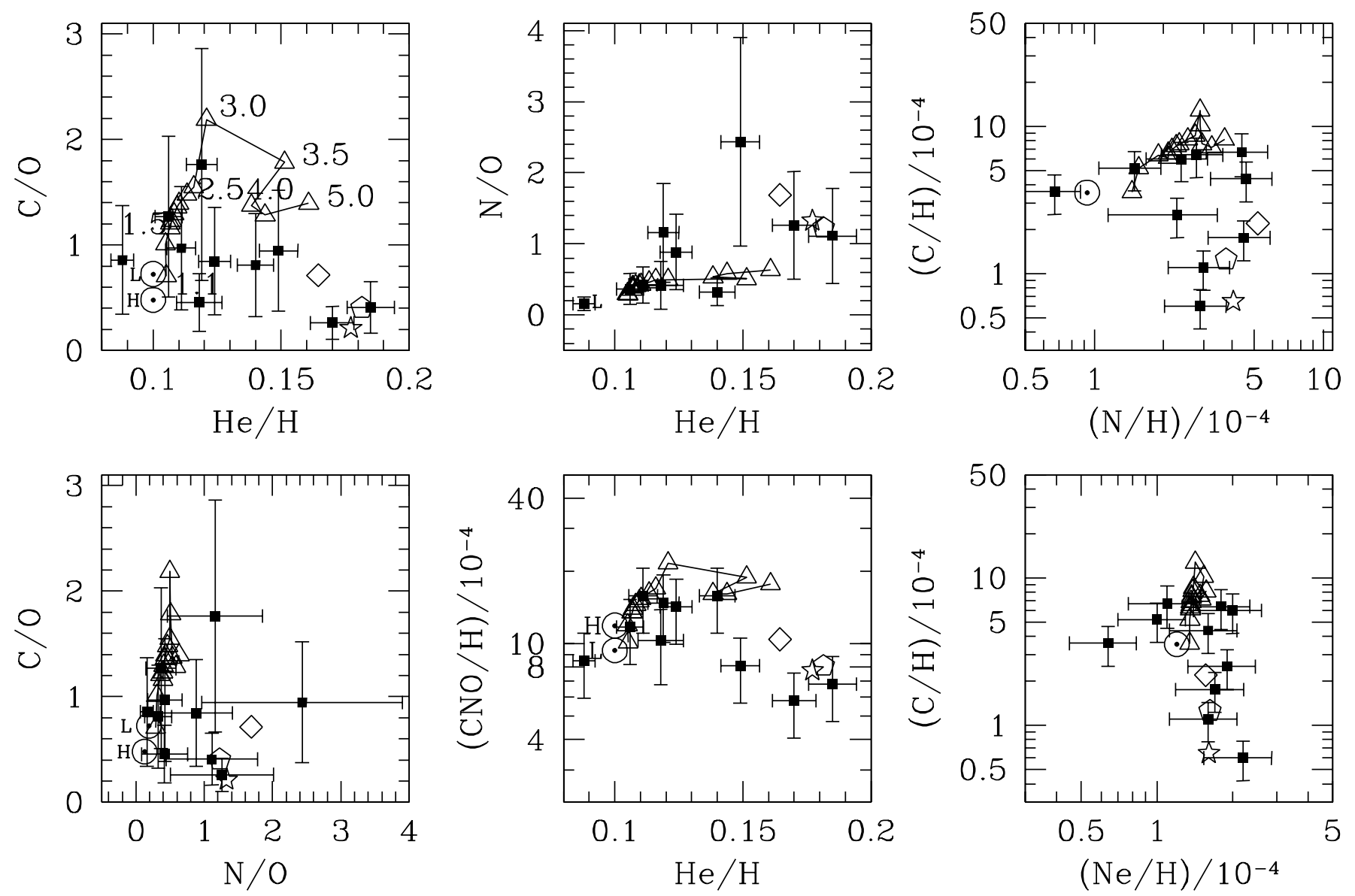

Fig. 12. The same as in Fig. 11, but expressing the abundance data with different combinations of elemental ratios.

expected abundance variations during the TP-AGB phase of intermediate-mass stars and the observed $\mathrm{PNe}$ abundances (see their Sect. VII). These authors demonstrated that the efficiency of $\mathrm{HBB}$, required to achieve the small $\mathrm{C} / \mathrm{O}$ values exhibited by $\mathrm{PNe}$ with large $\mathrm{He} / \mathrm{H}$, is such as to yield $\mathrm{N} / \mathrm{O}$ ratios that exceed by over an order of magnitude the PN values. This indication is quite robust and almost model-independent, since it merely reflects the interplay between nuclear reactions of the $\mathrm{CN}$-cycle. The efficiency of the third dredge-up directly affects the HBB nucleosynthesis: if the $\mathrm{CN}$-cycle operates at equilibrium (a condition often met by stars with HBB) the more carbon that is dredged-up, the more nitrogen is eventually synthesised.

After discussing various aspects of the issue, Becker \& Iben (1980) concluded that the positive correlation between N/O and $\mathrm{He} / \mathrm{H}$ observed in helium-rich $\mathrm{PNe}$ could be reproduced "by supposing that ${ }^{12} \mathrm{C}$ is burned at a modest rate in the convective envelope" of the most massive TP-AGB stars. In other words, they invoked a weak efficiency of HBB. However, these authors also clearly stated that in this way one would at the same time cope with the discrepancy of predicting too large atomic $\mathrm{C} / \mathrm{O}$ ratios, contrary to observations. The only possible explanation, plausible at that time, to reconcile all points was the hypothesis of a significant dust-depletion of carbon, with consequent apparent decrease of the measured $\mathrm{C} / \mathrm{O}$ ratio (involving the atomic carbon). In this study we explore other possibilities, as reported below.
The effect of variable molecular opacities. First we consider the effect of variable molecular opacities. Replacing the $\kappa_{\text {fix }}$ with $\kappa_{\text {var }}$ prescription in the $5 M_{\odot}$ model (while keeping $\lambda=0.9$, and the standard chemical composition), the evolution of $\mathrm{He}, \mathrm{C}, \mathrm{N}$ surface abundances change dramatically. In practice, we pass from the problem of a huge nitrogen over-production for the $\kappa_{\text {fix }}$ case (dot-long-dashed N/H curve) to those of almost zero nitrogen synthesis and carbon overenrichment for the $\kappa_{\mathrm{var}}$ model (short-long-dashed line).

The latter result is due to the weakening, or even prevention, of HBB in intermediate-mass stars that undergo efficient carbon enrichment by the third dredge-up during the early stages of their TP-AGB evolution. As discussed by Marigo (2003), the increase in molecular opacities, as soon as C/O becomes larger than one, causes cooling at the base of the convective envelope, which may extinguish the $\mathrm{CNO}$-cycle reactions associated with $\mathrm{HBB}$.

Both models with different opacities do not reproduce the observed PN data for $\mathrm{C}$ and $\mathrm{N}$, though both seem to imply the same direction: too much carbon is assumed to be injected by the third dredge-up into the convective envelope. This causes the over-production of nitrogen in the $\kappa_{\text {fix }}$ model, while it yields a net over-enrichment of carbon in the $\kappa_{\mathrm{var}}$ model.

These considerations introduce us to another possibility to solve this problem, related to the chemical composition of the convective inter-shell. 
Inter-shell chemical composition. We propose here a solution, different from that suggested by Becker \& Iben (1980), to account simultaneously for the observed N/O and $\mathrm{He} / \mathrm{H}$ correlation and $\mathrm{C} / \mathrm{O}$ and $\mathrm{He} / \mathrm{H}$ anti-correlation. It is based on the assumed elemental abundances - essentially ${ }^{4} \mathrm{He},{ }^{12} \mathrm{C}$, and ${ }^{16} \mathrm{O}$ - in the convective inter-shell developed at thermal pulses, part of which is then dredged-up to the surface.

We find that all discrepancies - relative to the $\mathrm{N}$ and/or $\mathrm{C}$ over-production - are removed by relaxing the usual prescription of the standard inter-shell chemical composition (Boothroyd \& Sackmann 1998, see also Sect. 5.1, and Table 4), and assuming instead that the dredged-up material in intermediate-mass TP-AGB stars consists mainly of helium, with very little carbon and practically no oxygen. Results are shown in Fig. 10 (solid, short- and long-dashed curves). Thanks to the small amount of dredged-up carbon, we would favour the enrichment in helium and avoid the nitrogen over-production.

These indications have been initially derived from empirical evidence, and it is encouraging to receive also some theoretical support from calculations of the TP-AGB evolution of intermediate-mass stars. To our knowledge, it was first mentioned - that the typical chemical inter-shell composition may be quite different from the standard one in the most massive TP-AGB stars - in the work of Vassiliadis \& Wood (1993). The authors point out that the thermal pulses in TP-AGB stars as massive as $5 M_{\odot}$ are characterised by extremely deep dredgeup $(\lambda \approx 0.8)$, and weak efficiency of the triple- $\alpha$ reaction in the convective inter-shell due to both the rapid quenching of the instability. These authors state that in their calculations the dredged-up material mainly consists of helium and nitrogen produced by the CNO cycle. Consequently, this result should imply a lower synthesis of primary carbon in the convective inter-shell, hence a lower carbon abundance in the dredgedup material compared to the standard values $\left(X_{\mathrm{csh}}\left({ }^{12} \mathrm{C}\right)<\right.$ $0.2-0.3)$. This indication agrees with our suggestion to explain the measured $\mathrm{CNO}$ abundances in PNe with very high helium content.

A few years later, Frost et al. (1998) partly confirmed the results by Vassiliadis \& Wood (1993), and pushed the analysis further reporting the discovery of a new kind of thermal pulses, designated as "degenerate thermal pulses" (the reader should refer to that work for all details). In few words a massive TP-AGB star would experience a sort of cyclic trend: First a relatively large number of weak thermal pulses (say 30-40) with very deep dredge-up takes place leaving a long tail of unburned helium, that is then burned at once by a strong "degenerate pulse" (the carbon abundance in the inter-shell reaches ${ }^{12} \mathrm{C} \approx 0.6$ ), after which another sequence of weak pulses starts again, and so on. Similar trends have been reported by Siess et al. (2002) in their study of the TP-AGB evolution in population III stars.

It should be remarked that while there is a close agreement in the results by Frost et al. (1998) and Vassiliadis \& Wood (1993) with respect to the high efficiency of the third dredge-up $(\lambda \approx 1)$ in these massive AGB stars, some substantial differences could be present instead in the predicted chemical composition of the dredged-up material. In fact, the possibility of a carbon abundance lower than the standard value
$\left(X_{\mathrm{csh}}\left({ }^{12} \mathrm{C}\right)<0.2-0.3\right)$, which is deduced from Vassiliadis \& Wood's paper, is actually not confirmed by the work of Frost et al. (1998; also Lattanzio's private communication). These possible differences could be ascribed to different physical and numerical details of the stellar evolution codes, that can significantly affect the results (see Frost et al. 1998; Frost \& Lattanzio 1996; and Lattanzio's private communication).

We believe that this point is crucial and it deserves a clarification with the aid of full calculations of the thermal pulses experienced by the most massive AGB models. Anyway, as our study represents a sort of empirical calibration of the AGB nucleosynthesis, we follow Vassiliadis \& Wood's indications and assume the possibility that during their TP-AGB evolution the most massive intermediate-mass stars suffer many deep dredgeup events that bring a large amount of helium, but little carbon up to the surface. In fact, as mentioned above, this seems just what we need to reproduce the observed abundances of the most He-rich PNe, thus solving a long-standing problem already stated by Becker \& Iben (1980).

The quantitative confirmation comes from the results of our TP-AGB calculations presented in Fig. 10, referring to models with i) stellar masses in the range $4.5-5.0 M_{\odot}$, ii) initial LMC composition $(Z=0.008)$, iii) assumed very deep dredgeup $(\lambda \sim 0.9)$, and iv) inter-shell chemical composition with typically $\left(X_{\mathrm{csh}}\left({ }^{12} \mathrm{C}\right)=0.02-0.03, X_{\mathrm{csh}}\left({ }^{16} \mathrm{O}\right)=0.002-0.003\right.$, $\left.X_{\text {csh }}\left({ }^{4} \mathrm{He}\right)=0.967-0.978\right)$. These models experience also HBB (more efficient at larger masses) as we see by considering the mirror-like evolution of the $\mathrm{C}$ - and $\mathrm{N}$-curves in the top-left and top-right panels, respectively. The final points, which would correspond to the predicted PN abundances, are clearly in very good agreement with the observed data for the most He-rich $\mathrm{PNe}$. In particular, models are able to reach $\mathrm{He} / \mathrm{H} \sim 0.17-0.20$, without over-producing nitrogen, and accounting for the extent of carbon depletion. We note that the rising part of the C-curves towards the end of the evolution, and the concomitant flattening of the $\mathrm{N}$-curves reflect the eventual extinction of $\mathrm{HBB}$ while the last dredge-up events still take place.

\subsubsection{Constraints from neon abundances}

Within the lowest extension of the error-bars, the data for the most He-rich $\mathrm{PNe}$ do not show a significant overabundance with respect to solar, though some increasing trend of $\mathrm{Ne} / \mathrm{H}$ with $\mathrm{He} / \mathrm{H}$ might be present. Some useful indications can be derived.

Recalling that both the first and second dredge-up episodes are expected not to alter the initial neon abundance, and looking at Fig. 4 we point out two facts, namely: i) the predicted $\mathrm{Ne} / \mathrm{H}$ after the second dredge-up in the most massive stars (with $M \sim 4-5 M_{\odot}$ ) with initial solar composition are already compatible with the measured $\mathrm{Ne} / \mathrm{H}$ in the most He-rich $\mathrm{PNe}$, whereas the models of the same stellar mass but with LMC composition lie clearly below the observed points; ii) the slightly increasing trend of $\mathrm{Ne} / \mathrm{H}$ with $\mathrm{He} / \mathrm{H}$ at increasing stellar mass merely reflect the decrease in the surface $\mathrm{H}$ content due to the dredge-up events, since the neon abundance is completely unaffected. 
The consideration of these two points, together with the conclusions from the analysis carried out in the preceding sections, would point to a significant Ne production having occurred in the progenitor stars of the most He-rich PNe, since all other PN abundances ( $\mathrm{He}, \mathrm{C}, \mathrm{N}$, and $\mathrm{O}$ ) seem globally consistent with stars originating from gas of sub-solar metallicity. As shown, our intermediate-mass models with LMC composition do satisfy all these chemical constraints.

Of course, another possibility would be $(\mathrm{Ne} / Z)_{\mathrm{LMC}}>$ $(\mathrm{Ne} / Z)_{\odot}$, that is the initial content of $\mathrm{Ne}$ in the original metalpoor gas was enhanced compared to what is expected for a solar-metallicity-scaled mixture. In this case, there is no necessity that $\mathrm{Ne}$ is synthesised during the AGB phase.

Going back to the former alternative, which invokes a sizeable Ne production in intermediate-mass stars with LMC composition, we find that models are able to attain a good agreement with the $\mathrm{Ne} / \mathrm{H}$ values measured in the most He-rich $\mathrm{PNe}$ by assuming that the enrichment of neon is due to the synthesis of ${ }^{22} \mathrm{Ne}$ during thermal pulses via the chain of reactions ${ }^{14} \mathrm{~N}(\alpha, \gamma){ }^{18} \mathrm{~F}\left(\beta^{+}, v\right){ }^{18} \mathrm{O}(\alpha, \gamma){ }^{22} \mathrm{Ne}$. In other words, all nitrogen in the inter-shell should be converted into ${ }^{22} \mathrm{Ne}$ (see Sect. 5.1). The possible channel of subsequent destruction through ${ }^{22} \mathrm{Ne}(\alpha, n){ }^{25} \mathrm{Mg}$ should be inefficient. In our calculations we assume that just $1 \%$ of the newly synthesised ${ }^{22} \mathrm{Ne}$ is burned into ${ }^{25} \mathrm{Mg}$ (see also Sect. 5.1).

Then, in the context of the proposed interpretation, the immediate consequence would be the inefficiency of the ${ }^{22} \mathrm{Ne}(\alpha, n){ }^{25} \mathrm{Mg}$ reaction as production channel of neutrons, with important implications for the synthesis of slow-neutron capture elements (Busso et al. 1999). In this case, the major role for the s-process nucleosynthesis would be played by the ${ }^{13} \mathrm{C}(\alpha, n){ }^{16} \mathrm{O}$ channel.

\section{Summary and conclusions}

In this study a sample of PNe with accurately determined elemental abundances, is used to derive interesting information about the evolution of the stellar progenitors, and set constraints on the nucleosynthesis and mixing processes characterising their previous evolution. To this aim, synthetic TP-AGB models are calculated to reproduce the data by varying the parameters: initial stellar mass and metallicity, molecular opacities, dredge-up and HBB efficiency, and chemical composition of the convective inter-shell developed at thermal pulses.

The clear segregation of the abundance data in two subsamples, particularly evident in the $\mathrm{O} / \mathrm{H}-\mathrm{He} / \mathrm{H}$ diagram, has led us to discuss them separately. And indeed, our investigation suggests two different interpretative scenarios. The final results that best reproduce the observed data are summarised in Figs. 11 and 12.

From the analysis of the group of PNe with low He content $(\mathrm{He} / \mathrm{H}<0.15)$ and solar-like oxygen abundances, we conclude that:

- The stellar progenitors are low- and intermediate-mass stars with original solar-like chemical composition and initial masses spanning the range $0.9-4.0 M_{\odot}$.
- The oxygen abundances are consistent with the recent determination for the Sun by Allende Prieto et al. (2001), that is lower than previous estimates (see e.g. Anders \& Grevesse 1989) by almost 0.2 dex. For a few PNe there may be a limited oxygen enrichment, possibly associated with dredge-up during the TP-AGB phase.

- There is clear evidence of carbon enrichment in some PNe that also exhibit $\mathrm{C} / \mathrm{O}>1$, suggesting that they evolved from carbon stars experiencing the third dredge-up during the TP-AGB phase.

- Measured carbon abundances are well reproduced by TP-AGB models with dredge-up efficiencies $\lambda \sim 0.3-0.4$, and adopting variable molecular opacities in place of the usual solar-scaled opacity tables (see Marigo 2002). The introduction of variable opacities prevents the likely overenrichment of carbon by shortening the duration of the carbon-star phase, and causing an earlier shut-down of the third dredge-up due to the cooling of the envelope structure (Marigo 2003).

- The degree of nitrogen enrichment is consistent with the expectations from the first and second dredge-up events, occurred prior to the TP-AGB phase. The efficiency of HBB in intermediate-mass stars with solar-metallicity should be modest.

- Helium abundances are well accounted for by considering the whole contribution of all dredge-up processes (i.e. first, and possibly second and third).

From the study of the extremely helium-rich $(0.15 \leq \mathrm{He} / \mathrm{H} \leq$ 0.20 ) and oxygen-poor PNe we can conclude the following:

- The stellar progenitors should be intermediate-mass stars (4-5 $M_{\odot}$ ) experiencing both the third dredge-up and HBB during their TP-AGB evolution.

- The PN oxygen abundances are consistent with a sub-solar initial stellar metallicity. In fact, under the hypothesis of solar metallicity we are forced to invoke a significant oxygen destruction via very efficient $\mathrm{HBB}$, which violates other chemical constraints, e.g. causing a large over-production of nitrogen. Instead, models with assumed initial LMC composition provide a fairly good agreement with the data.

- The first two assumptions are needed in the models to reproduce the observed abundances. This leads to a controversy which is important to point out. The combination of low metallicity with intermediate-mass progenitors is peculiar, since these stars are probably recently formed from gas with interstellar abundances. Although there are several indications that these PNe are of lower metallicity (see Sect. 6.4.1), this could also suggest that perhaps another physical process has not fully been taken into account. This issue should be further investigated.

- The long-standing problem - initially formulated by Becker \& Iben (1980) - of accounting, simultaneously and quantitatively, for the observed N/O-He/H correlation and the $\mathrm{C} / \mathrm{O}-\mathrm{N} / \mathrm{O}$ anti-correlation seems to be solved by assuming that the third dredge-up i) is very efficient, and ii) brings up to the surface material containing only a small amount of primary carbon synthesised during thermal pulses. A good 
agreement with the observed data is obtained by adopting $\lambda \sim 0.9$ and $X_{\mathrm{csh}}\left({ }^{12} \mathrm{C}\right) \sim 0.02-0.03$.

The former indication on the dredge-up efficiency, derived empirically, is supported on theoretical grounds by full TP-AGB calculations of intermediate-mass stars, i.e. Vassiliadis \& Wood (1993), and more recently Frost et al. (1998) and Siess et al. (2002). A significant part of the helium enrichment of these PNe should be ascribed to a large number of deep dredge-up events that precede the occurrence of the so-called "degenerate pulses", according to the designation introduced by Frost et al. (1998). The additional requirement emerging from our study - that such dredge-up events should not only be extremely deep but also carry a small amount of carbon - is not fully confirmed by theoretical analyses (i.e. Frost et al. 1998), though a positive indication in this sense is given by the work of Vassiliadis \& Wood (1993).

- A significant production of ${ }^{22} \mathrm{Ne}$ - via $\alpha$-captures starting from ${ }^{14} \mathrm{~N}$ - should take place in these stars to reproduce the observed $\mathrm{Ne} / \mathrm{H}$ values of the $\mathrm{He}$-rich $\mathrm{PNe}$, under the hypothesis they descend from intermediate-mass stars with initial LMC chemical composition. As direct consequence, this would imply a reduced role of the ${ }^{22} \mathrm{Ne}(\alpha, n)^{25} \mathrm{Mg}$ reaction in providing neutrons for the slow-neutron capture nucleosyntesis that is expected to occur during thermal pulses. We also note that the invoked inefficiency of the ${ }^{22} \mathrm{Ne}(\alpha, n){ }^{25} \mathrm{Mg}$ channel seems consistent with the expected low synthesis of carbon at thermal pulses in the most massive AGB stars experiencing very deep dredge-up (see former point). In fact, as reported by Vassiliadis \& Wood (1993) the deep dredge-up quickly extinguishes the helium burning shell. As a consequence this could prevent both a significant production of carbon via the triple- $\alpha$ reaction and the attainment of the high temperatures required for the full activation of the ${ }^{22} \mathrm{Ne}(\alpha, n)^{25} \mathrm{Mg}$ reaction. The confirmation of this two-fold aspect deserves detailed calculations of thermal pulses.

Acknowledgements. P.M. acknowledges the SRON National Institute for Space Research (Groningen) for hospitality and financial support during her visit in October 2001, and the Italian Ministry of Education, University and Research (MIUR) for the work carried out at the Astronomy Department in Padova. J. Bernard-Salas thanks Annette Ferguson for valuable discussions and suggestions as well as the Università di Padova for hospitality. We are grateful to Dr. John Lattanzio for the careful reading of this manuscript, and for providing comments and suggestions that result in an improvement of the paper.

\section{References}

Acker, A., Ochsenbein, F., Stenholm B., et al. 1992, Catalogue of Galactic Planetary Nebulae-Part II, Strasbourgh-ESO Lambert, D.L., \& Asplund, M. 2001, ApJ, 556, L63

Alexander, D. R., \& Ferguson, J. W. 1994, ApJ, 437, 879

Allende Prieto, C., Lambert, D. L., \& Asplund, M. 2001, ApJ, 556, 63
Anders, E., \& Grevesse, N. 1989, Geochim. Cosmochim. Acta, 53, 197

Bains, I., Bryce, M., Mellema, G., Redman, M. P., \& Thomasson, P. 2003, MNRAS, 340, 381

Becker, S. A., \& Iben, I., Jr. 1980, ApJ, 237, 111

Beintema, D. A., \& Pottasch, S. R. 1999, A\&A, 347, 942

Bernard Salas, J., Pottasch, S. R., Beintema, D. A., \& Wesselius, P. R. 2001, A\&A, 367, 949

Bernard Salas, J., Pottasch, S. R., Feibelman, W. A., \& Wesselius, P. R. 2002, A\&A, 387, 301

Bernard-Salas, J., Pottasch, S. R., Wesselius, P. R., \& Feibelman, W. A. 2003a, A\&A, 406, 165

Bernard-Salas, J., Pottasch, S. R., Beintema, D. A., \& Wesselius, P. R. 2003b, A\&A, 406, 175

Boothroyd, A. I., \& Sackmann, I.-J. 1988, ApJ, 328, 653

Busso, M., Gallino, R., \& Wasserburg, G. J. 1999, ARA\&A, 37, 239

Caughlan, G. R., \& Fowler, W. A. 1988, Atomic Data Nucl. Data Tables, 40, 283

Charbonnel, C. 1995, ApJ, 453, L41

Ciardullo, R., Bond, H. E., Sipior, M. S., et al. 1999, AJ, 118, 488

Corradi, R. L. M., \& Schwarz, H. E. 1993, A\&A, 269, 462

Dopita, M. A., Vassiliadis, E., Wood, P. R., et al. 1997, ApJ, 474, 188

Edvardsson, B., Andersen, A., Gustafsson, B., et al. 1993, A\&A, 275, 101

Esteban, C., Peimbert, M., Torres-Peimbert, S., \& Escalante, V. 1998, MNRAS, 295, 401

Forestini, M., \& Charbonnel, C. 1997, A\&AS, 123, 241

Frost, C. A., \& Lattanzio, J. C. 1996, ApJ, 473, 383

Frost, C. A., Lattanzio, J. C., \& Wood, P. R. 1998, ApJ, 500, 355

Girardi, L., Bressan, A., Bertelli, G., \& Chiosi, C. 2000, A\&AS, 141, 371

Grevesse, N., \& Noels, A. 1993, in Origin of the Elements, ed. N. Prantzos et al. (Cambridge University Press), 15

Grevesse, N., \& Sauval, A. J. 1998, Space Sci.Rev., 85, 161

Groenewegen, M. A. T., van den Hoek, L. B., \& de Jong, T. 1995, A\&A, 293, 381

Heap, S. R., Corcoran, M., Hintzen, P., \& Smith, E. 1989, in From Miras to Planetary Nebulae, ed. M. O. Mennessier, \& A. Omont (Éditions Frontières), 397

Henry, R. B., Kwitter, K. B., \& Bates, J. A. 2000, ApJ, 531, 928

Herwig, F., Blöcker, T., Schönberner, D., \& El Eid, M. 1997, A\&A, 324, L81

Hyung, S. 1999, JKAS, 32, 55

Iben, I., \& Renzini, A. 1983, ARA\&A, 21, 271

Karakas, A. I., Lattanzio, J. C., \& Pols, O. R. 2002, PASA, 19, 515

Kessler, M. F., Steinz, J. A., Anderegg, M. E., et al. 1996, A\&A, 315, L27

Kingsburgh, R. L., \& Barlow, M. J. 1994, MNRAS, 271, 257

Kilian-Montenbruck, J., Grehen, T., \& Nissen, P. E. 1994, ApJ, 291, 757

Lame, N. J., \& Ferland, G. J. 1991, ApJ, 367, 208

Liu, X.-W., Barlow, M. J., Cohen, M., et al. 2001, MNRAS, 323, 343

Marigo, P. 1998, A\&A, 340, 463

Marigo, P. 2001, A\&A, 370, 194

Marigo, P. 2002, A\&A, 387, 507

Marigo, P. 2003, in CNO in the Universe, PASP, ed. C. Charbonnel, D. Schaerer, \& G. Meynet, in press [astro-ph/0212261]

Marigo, P., Bressan, A., \& Chiosi, C. 1996, A\&A, 313, 545

Marigo, P., Bressan, A., \& Chiosi, C. 1998, A\&A, 331, 564

Marigo, P., Girardi, L., \& Bressan, A. 1999, A\&A, 344, 123

Martín-Hernández, N. L., Peeters, E., Morisset, C., et al. 2002, A\&A, 381,606

Meaburn, J., \& Walsh, J. R. 1989, A\&A, 223, 277 
Peimbert, M., \& Torres-Peimbert, S. 1987, Rev. Mex. Astron. Astrof., 14,540

Pottasch, S. R. 1984, Planetary Nebulae (D-Reidel), vol. 107

Pottasch, S. R. 2000, A\&A, 362, L17

Pottasch, S. R., \& Beintema, D. A. 1999, A\&A, 347, 975

Pottasch, S. R., Beintema, D. A., \& Feibelman, W. A. 2000, A\&A, 363,767

Pottasch, S. R., Beintema, D. A., Bernard Salas, J., \& Feibelman, W. A. 2001, A\&A, 380, 684

Pottasch, S. R., Beintema, D. A., Bernard Salas, J., Koornneef, J., \& Feibelman, W. A. 2002, A\&A, 393, 285
Reed, D. S., Balick, B., \& Hajian, A. R. 1999, AJ, 118, 2430

Siess, L., Livio, M., \& Lattanzio, J. 2002, ApJ, 570, 329

Stasińska, G., \& Tylenda, R. 1986, A\&A, 155, 137

Terzian, Y. 1997, IAU Symp. 180, 29

van Hoof, P. A. M., van de Steene, G. C., Beintema, D. A., et al. 2000, ApJ, 532, 384

Vassiliadis, E., \& Wood, P. R. 1993, ApJ, 413, 641

Vassiliadis, E., \& Wood, P. R. 1994, ApJS, 92, 125

Wood, P. R. 1981, in Physical processes in red giants, Proc. of the Second Workshop, Erice, Italy, September 3-13, 1980 (Dordrecht: D. Reidel Publishing Co.), 135 